Department of Diagnostic Radiology Helsinki University Central Hospital University of Helsinki, Finland

\title{
SPIN LOCK AND MAGNETIZATION TRANSFER IMAGING OF HEAD AND NECK TUMORS
}

Antti Markkola

Academic Dissertation

To be presented with the permission of the Faculty of M edicine of the University of Helsinki, for public discussion in Auditorium XIII

On October $31^{\text {th }}, 2003$, at 12 noon.

Helsinki 2003 


\section{Supervised by}

Docent Hannu Aronen, M.D., Ph.D.

Functional Brain Imaging Unit

Helsinki Brain Research Center

Professor Raimo Sepponen, Ph.D.

Faculty of Electrical Engineering

Helsinki University of Technology

\section{Reviewed by}

Docent Pekka Niemi, M .D.

Department of Radiology

University of Turku

M arkku Komu, Ph.D.

Department of Radiology

University of Turku

\section{To be discussed with}

Professor Ilkka Suramo, M .D.

Department of Radiology

University of Oulu

ISBN 952-91-6403-3 (nid.)

ISBN 952-10-1405-9 (PDF)

Yliopistopaino

Helsinki 2003 
To Helena and The Boys 



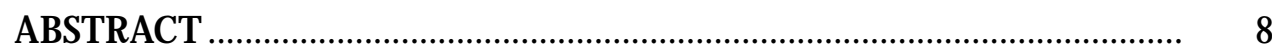

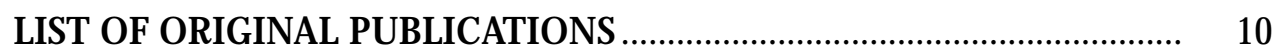

ABBREVIATIONS

INTRODUCTION

REVIEW OF THE LITERATURE ……................................................. 15

1. Principles of nuclear magnetic resonance ................................................. 15

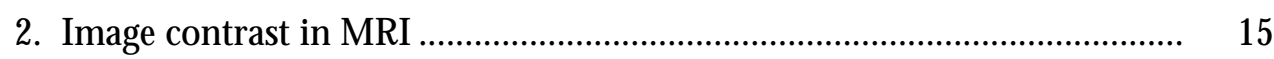

2.1 Contrast and contrast-to-noise......................................................... 16

2.2 Spin echo imaging ........................................................................ 16

2.3 Gradient echo imaging .................................................................. 16

2.3.1 GRE versus SE ................................................................... 17

2.3.2 Chemical shift based opposed-phase imaging ......................... 17

2.4 The M RI preparation pulses to modify image contrast ...................... 17

2.4.1 The inversion recovery techniques............................................ 18

2.4.2 Diffusion weighted imaging .................................................... 18

$2.5 \mathrm{Head}$ and neck M RI protocol for optimized detection of lesions....... 19

3. Tissue characterization in conventional M RI ............................................ 19

3.1 T1 and T2 relaxation times and tissue specificity............................. 19

3.2 Tissue characterization in head and neck tumor imaging ................... 20

3.2.1 Lymph node imaging studies ................................................ 20

3.2.1.1 Conventional methods ............................................... 20

3.2.1.2 Ultrasmall superparamagnetic iron oxide ................... 21

4. Other imaging methods for enhanced tissue specificity ............................ 22

4.1 Positron emission tomography ............................................................. 22

4.1.1 Evaluation of recurrent tumors................................................... 22

4.1.2 Unknown primary and nodal staging ...................................... 22

4.2 Radioimmunoscintigraphy and molecular markers .......................... 23

5. Magnetization transfer ........................................................................... 23

5.1 The concept of magnetization transfer and MT effect....................... 23

5.2 MT in molecular level.................................................................. 24

5.3 Tissue characterization with MT …................................................... 25 
5.4 Tissue characterization of head and neck and brain tumors with MT 25

5.5 MT and Contrast improvement..................................................... 26

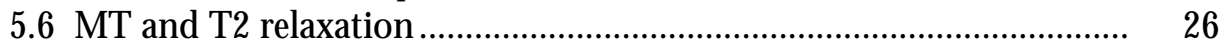

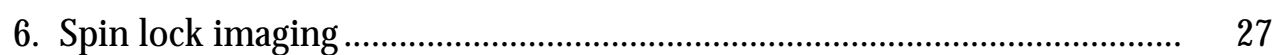

6.1 SL technique............................................................................ 27

6.2 Evaluation of contrast with SL imaging .......................................... 28

6.3 SL imaging and tissue characterization ............................................. 28

6.4 Comparison between SL and MT imaging ......................................... 29

$6.5 \mathrm{~T} 1 \rho$ dispersion imaging.................................................................... $\quad 29$

7. Low field versus high field .......................................................................... 30

7.1 The field dependence of relaxation and contrast ............................... $\quad 30$

7.2 Clinical imaging and field strength .................................................... 31

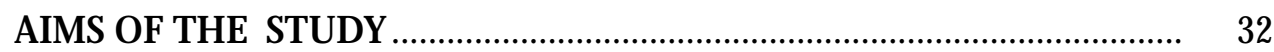

MATERIALS AND METHODS

1. Patients and normal volunteers........................................................... 33

2. M R imaging protocols

2.1 Single slice SL sequence.................................................................... 33

2.2 Multiple slice SL sequence............................................................... 34

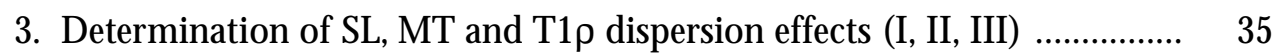

4. Relaxation time measurements (III, IV) ................................................... 36

$4.1 \mathrm{~T} 1$ and T2 measurements ............................................................... 36

$4.2 \mathrm{~T} 1 \rho$ measurements .................................................................... 36

5. Exploring multiple slice SL imaging parameters (IV) ............................... 36

6. Comparison of tumor CNR between SL and T2-weighted sequences (IV) 37

7. Pathologic evaluation ..................................................................... 37

8. Statistical methods …............................................................................. 37

RESULTS

1. Head and neck tissue characterization $(\mathrm{I}, \mathrm{II}, \mathrm{III})$........................................ 39

$1.1 \mathrm{SL}$ and MT effects of tumors $(\mathrm{I}, \mathrm{II})$................................................... 39

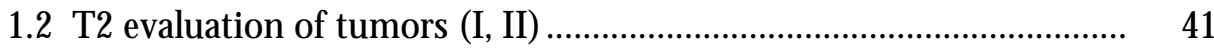

$1.3 \mathrm{SL}$ and MT effects of normal tissues (I) ........................................... 41

1.4 Tlp dispersion imaging of tumors (II) ............................................. 42

$1.5 \mathrm{~T} 1 \rho$ dispersion effects of normal tissues $(\mathrm{II}, \mathrm{III})$................................ 42 
2. The relaxation time measurements of different tissues and tumors (III, IV)

3. Evaluation of multiple slice SL imaging parameters (IV)

4. Comparison of tumor CNR between SL and T2-weighted sequences (IV)

DISCUSSION

1. Determination of relaxation parameters for head and neck tissues (III, IV)

2. Multiple slice SL technique (IV) ............................................................

2.1 Evaluation of imaging parameters ...................................................... 48

2.2 Evaluation of contrast.................................................................... 49

2.2.1 Comparison of tumor CNR between SL and T2-weighted sequences

2.2.2 Salivary gland opposed-phase multiple slice SL imaging......... $\quad 49$

2.2.3 Comparison between SL- and T2-weighted imaging techniques.. $\quad 50$

2.2 .4 Fat ........................................................................... 50

2.2.5 GRE- versus SE-SL imaging ............................................ $\quad 50$

3. Head and neck tissue characterization $(\mathrm{I}, \mathrm{II})$.......................................... 51

3.1 T2 evaluation versus SL and M T effects........................................ 51

3.2 Tumor characterization with M T and SL effects ............................... 51

3.3 Correlation and efficiency of SL and MT techniques ....................... 53

3.4 Single slice and multiple slice imaging techniques and tissue characterization ......................................................................... 53

3.5 References $\mathrm{M} \mathrm{nCl}$ and fat ......................................................... 54

3.6 Tlp dispersion imaging of head and neck tissues ............................. 54

4. Future prospects ................................................................................ 55

5. Clinical implications ...................................................................... 56

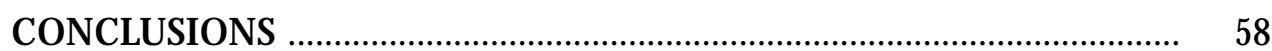

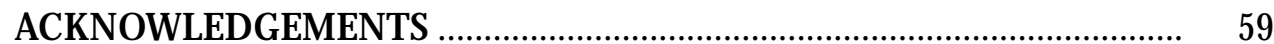

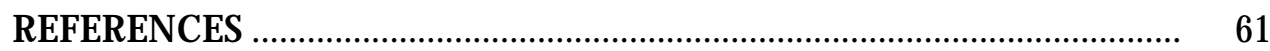

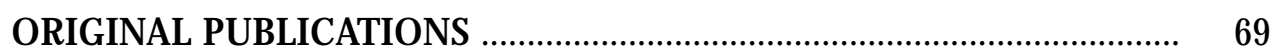




\section{SPIN LOCK AND MAGNETIZATION TRANSFER IMAGING OF HEAD AND NECK TUMORS}

Background: Due to high soft tissue contrast magnetic resonance imaging (M RI) is often the method of choice in the staging of head and neck neoplasms. The problem with conventional M RI is its relatively poor capability to predict the tissue composition of a lesion. In some M R imaging techniques such as magnetization transfer (MT) and spin lock (SL), the image contrast has been shown to be related to macromolecular composition of tissues. These techniques have been applied for tumor characterization and demonstrated to have enhanced tissue contrast for a variety of diseases. The purpose of this thesis was to develop multiple slice SL sequences for head and neck tumor imaging at $0.1 \mathrm{~T}$ and to compare the tumor detection capability of SL technique with conventional T2-weighted technique. The tumor characterization potential of SL and MT techniques was also evaluated.

Methods: In all, fifty-three patients with histologically or cytologically confirmed head and neck tumors and sixteen healthy volunteers were studied with a $0.1 \mathrm{~T}$ $\mathrm{M} \mathrm{R}$ device. The T1 $\rho, \mathrm{T} 1$ and $\mathrm{T} 2$ relaxation times for different head and neck tissues and tumors were determined by fitting the measured signal intensities to the corresponding equations. The relaxation times were used to optimize contrast-tonoise ratios (CNR) between pairs of tumor (epidermoid carcinoma) and different head and neck tissues. The effect of changing different imaging parameters on image CNR was evaluated in order to determine the optimal sequence parameters for head and neck tumor imaging. In patient study, the tumor CNR in multiple slice SL-GRE (1500/30 ms) images was compared with conventional spin echo (SE) T2-weighted (1500/30/120 ms) images. The signal intensity reduction caused by SL, T1 $\rho$ dispersion or MT preparation pulse (SL effect, T1 $1 \rho$ dispersion effect or MT effect) was determined and evaluated in the differentiation of benign from malignant head and neck tumors.

Results: T1 $1 \rho$ values of tumors and normal head and neck tissues, measured at locking pulse amplitude B1L of $35 \mu \mathrm{T}$, were slightly longer than corresponding $\mathrm{T} 2$ relaxation times. The exception was fat which had considerably longer $\mathrm{T} 1 \rho$ than T2. For salivary gland tumors the mean CNR was significantly greater with the opposed phase SL-GRE technique than with the T2-weighted SE technique (1.2 \pm 0.6 vs. $0.8 \pm 0.6, p=0.03$, respectively, $t$ test). The mean CNR for tumors was similar on SL-GRE images compared to T2-weighted SE images ( $1.1 \pm 0.8$ vs. 1.0 $\pm 0.8, p=0.5$, respectively, $t$ test). A good correlation ( $r=0.64, p<0.001)$ in tumor CNRs between SL images and T2-weighted SE images was found. Com- 
parison of the measured SL and MT effects in head and neck tumors showed strong correlation $(r=0.85, p<0.001)$ between these modalities. With an SL effect of 0.48 and an MT effect of 0.32 as the threshold, the sensitivity for detecting a malignant tumor was $96 \%$ and $90 \%$, specificity $67 \%$ and $71 \%$, and accuracy $81 \%$ and $81 \%$ for SL effect and M T effect, respectively.

Conclusions: Multiple slice SL-GRE technique provides image contrast comparable to SE T2-weighted imaging for head and neck tumors. The combination of short locking pulses (TL $=10-35 \mathrm{~ms}$ ) and of short TE of $30 \mathrm{~ms}$ produces optimum CNR for clinical tumor imaging with wide anatomical coverage and re duced motion and susceptibility artifacts compared to T2-weighted imaging. The out of phase fat/water SL technique is especially advantageous in salivary gland tumor imaging. Strong correlation between SL and MT effects in head and neck tumors supports the theory that magnetization transfer is a dominant relaxation mechanism in SL technique in protein rich tissues. Low T1 1 dispersion effects, SL effects and MT effects are characteristic of benign head and neck tumors. High effect values are not specific indicators of malignancy because effects of chronic salivary gland infections and some benign tumors and malignancies may overlap. 


\section{LIST OF ORIGINAL PUBLICATIONS}

This thesis is based on the following publications and in the text they are referred to as their Roman numerals I-IV.

I Markkola AT, Aronen HJ, Paavonen T, Hopsu E, Sipilä LM, Tanttu JI, Sepponen RE. Spin lock and magnetization transfer imaging of head and neck tumors. Radiology 1996; 200:369-375

II Markkola AT, Aronen HJ, Paavonen T, Hopsu E, Sipilä LM, Tanttu JI, Sepponen RE. T1p dispersion imaging of head and neck tumors: a comparison to spin lock and magnetization transfer techniques. J Magn Reson Imaging $1997 ; 7: 873-879$

III Markkola AT, Aronen HJ, Abo Ramadan U, Halavaara JT, Tanttu JI, Sepponen RE. Determination of T1 $\rho$ values for head and neck tissues at 0.1 $\mathrm{T}$ : a comparison to $\mathrm{T} 1$ and $\mathrm{T} 2$ relaxation times. Magn Reson Imaging 1998;16:377-383

IV Markkola AT, Aronen HJ, Lukkarinen S, Abo Ramadan U, Tanttu JI, Sepponen RE. Multiple-slice spin lock imaging of head and neck tumors at $0.1 \mathrm{~T}$ : Exploring appropriate imaging parameters with reference to T2-weighted spin echo. Invest Radiol 2001;36:531-538

Permission for reprinting was requested from the Publishers. 


\section{ABBREVIATIONS}

\begin{tabular}{|c|c|}
\hline$\alpha$ & Flip angle \\
\hline ADC & Apparent diffusion coefficient \\
\hline $\mathrm{B}_{0}$ & Main magnetic field \\
\hline & $\begin{array}{l}\text { Amplitude of the excitatory radio frequency field or preparation } \\
\text { pulse }\end{array}$ \\
\hline B1L & Amplitude of the spin lock pulse \\
\hline & Effective magnetic field \\
\hline CNR & Contrast-to-noise ratio \\
\hline CT & Computed tomography \\
\hline DWI & Diffusion weighted imaging \\
\hline & Larmor frequency \\
\hline FA & Flip angle \\
\hline FDG & Fluorine labeled deoxyglucose \\
\hline FLAIR & Fluid attenuated inversion recovery \\
\hline FSE & Fast spin echo \\
\hline Gd-DTPA & Gadolinium diethylenetriamine penta-acetic acid \\
\hline GRE & Gradient echo \\
\hline $\mathrm{Hf}$ & Protons of freely moving water molecules \\
\hline $\mathrm{Hr}$ & Protons of restricted mobility associated with macromolecules \\
\hline IR & Inversion recovery \\
\hline K & Pseudo first-order rate constant of magnetization transfer \\
\hline$M_{0}$ & Equilibrium magnetization \\
\hline $\mathrm{MnCl}$ & M anganese chloride \\
\hline MRA & M agnetic resonance angiography \\
\hline MRI & Magnetic resonance imaging \\
\hline MT & Magnetization transfer \\
\hline MTC & Magnetization transfer contrast \\
\hline MT effect & Signal intensity reduction caused by M T preparation pulse \\
\hline MTR & Magnetization transfer ratio; same as M T effect \\
\hline $\mathrm{N}$ & Number of slices \\
\hline NMR & Nuclear magnetic resonance \\
\hline PET & Positron emission tomography \\
\hline ppm & parts per million \\
\hline$\rho$ & Density of mobile spins in the object \\
\hline RF & Radio frequency \\
\hline RIS & Radioimmunoscintigraphy \\
\hline ROI & Region of interest \\
\hline $\mathrm{S}$ & Signal intensity \\
\hline $\mathrm{S}_{0}$ & Equilibrium signal intensity \\
\hline SAR & Specific absorption rate \\
\hline SE & Spin echo \\
\hline
\end{tabular}


SL Spin lock

SL effect Signal intensity reduction caused by SL preparation pulse

SNR Signal-to-noise ratio

STIR Short tau inversion recovery

T Tesla

T1 Longitudinal relaxation time

T1p Longitudinal relaxation time in the rotating frame

$\mathrm{T} 1 \rho^{\text {off }} \quad$ Longitudinal relaxation time in the rotating frame in off-resonance experiment

T1sat Longitudinal relaxation time in the presence of magnetization transfer pulse

T2 Transverse relaxation time

T2* $\quad$ Transverse relaxation time combining the effects of $\mathrm{T} 2$ and susceptibility gradients and magnetic field inhomogeneities

Toff Duration of the magnetization transfer pulse

TD Repetition time of the locking pulses

TE Echo time

$\mathrm{TI} \quad$ Inversion time

TL Locking time

TR Repetition time

USPIO Ultrasmall superparamagnetic iron oxide 


\section{INTRODUCTION}

The appearance of tissue in magnetic resonance imaging (MRI) depends on inherent tissue properties and on extrinsic user selectable parameters. The most relevant tissue related parameters are $\mathrm{T} 1$ and $\mathrm{T} 2$ relaxation times and the proton density. The extrinsic user selectable parameters that affect the appearance of image include sequence parameters, preparation pulses and contrast agents. If one can determine the relaxation times for two tissues it is possible to optimize pulse sequence parameters so that the contrast between these tissues is maximized. However, the relaxation time measurements of tissue obtained with one type of M R imager are not directly comparable with data from another device, because of the imager specific systematic errors and because relaxation times are field strength dependent.

The lesion contrast is defined as the signal intensity difference between lesion and the surrounding tissue. However, when assessing the lesion conspicuity the image noise is an important factor to be considered. The image noise has been considered the primary obstacle for detection of low-contrast lesions in MRI (Edelstein et al.1983). In clinical imaging the contrast-to-noise ratio (CNR) of a lesion reflects the ability to detect pathological processes.

The preparation pulses have been developed to modify image contrast before the actual excitation pulse and data collection. Such preparation pulses include the fat-signal-suppression schemes, the inversion recovery (IR) techniques, and the tissue suppression concepts: the magnetization transfer (MT) (Wolff and Balaban 1989) and spin lock (SL) (Sepponen et al. 1985). In magnetization transfer and spin lock techniques a very low field RF pulse is applied before conventional imaging sequence, leading to variable suppression of tissues. In both techniques the resulting tissue suppression and image contrast are related to tissue macromolecule water interaction, and tissues with high macromolecular content have also strong signal intensity reduction (MT effect or SL effect). Both magnetization transfer and spin lock techniques have been used in a variety of occasions to enhance image contrast either with or without contrast medium administration.

The accurate characterization of a head and neck lesion is essential for choosing a suitable therapy for each patient. Tissue characterization in M RI has been conventionally related to relaxation time measurements of tissues and pathologies. Unfortunately, the results of such studies have not been encouraging (Bottomley et al, 1987). Furthermore, determination of relaxation times is a time consuming and technically demanding process, which is not practical for clinical imaging. MRI would have greater impact on patient management if a more reliable method to predict the histological nature of a head and neck tumor were developed. Determination of MT or SL effect is easy and fast to perform, with only two additional sequences to the MR imaging protocol. SL and MT effects have been used with promising results in the characterization of various pathologies. 
In this thesis, $\mathrm{T} 1 \rho, \mathrm{T} 1$ and $\mathrm{T} 2$ relaxation times for head and neck tissues and tumors were determined. Based on these parameter calculations multiple slice spin lock gradient echo sequences were developed and evaluated for head and neck tumor imaging, and the tumor CNR was compared with spin echo T2weighted images. The characterization potential of SL and MT effects in head and neck tumors was also evaluated. 


\section{Principles of nuclear magnetic resonance}

Nuclear magnetic resonance (N M R) was first demonstrated by Bloch et al. (1946) and Purcell et al. (1946). Since then N M R has had a great influence on chemistry, physics, material science, and later with the development of M RI, also on medicine. Lauterbur (1973) was the first to suggest the use of linear magnetic field gradients for obtaining images with NMR. As a result of this localization technique cross-sectional tomographic images in any direction could be obtained. Informative images for clinical applications did not become available until early eighties (Young et al.1981, Crooks et al. 1982).

Nuclei with an odd number of protons or neutrons or both are potentially applicable for MRI. These nuclei have a small angular momentum constantly spinning around an axis, called a spin. Because hydrogen nucleus consists of a single proton and is of great abundance in human tissues, it is an excellent element to be exploited for clinical imaging.

At zero magnetic field tissue protons have no net magnetization $\left(M_{0}\right)$. When exposed to static external magnetic field $\left(B_{0}\right)$ the protons align along the field and their spins start to precess around the axis of $B_{0}$ with a frequency proportional to the strength of the field, called the Larmor frequency $\left(\mathrm{f}_{0}\right)$. In equilibrium state greater numbers of protons occupy the lower energy state resulting in $M_{0}$, which is aligned along the $\mathrm{B}_{0}$ referred as $\mathrm{z}$-axis. In order to gain information from the object the spin system must be perturbed with a short temporary radio frequency (RF) pulse applied at the Larmor frequency. In RF field excitation the spins absorb energy and a transversal magnetic component is produced. After the RF pulse is switched off the nuclei return to the equilibrium state and emit RF radiation. The longitudinal relaxation time (T1) determinates the rate how fast the equilibrium state is achieved. The external magnetic field inhomogeneities and interaction between spins cause net magnetization to dephase in transverse plane. The rate of losing the phase coherence of spins is referred as the transverse relaxation time (T2). T2 decay depends primarily on spin-spin and spin-surroundings interactions whereas so called T2* decay depends additionally on susceptibility gradients and external polarizing field inhomogeneities.

\section{Image contrast in MRI}

The inherent tissue contrast in M RI is a function of $\mathrm{T} 1$ and $\mathrm{T} 2$ relaxation times and proton densities of tissues (Hendrick 1999). In addition, many other parameters including susceptibility, chemical shift, flow, perfusion and diffusion affect the appearance of the image. Extrinsic, user-selectable parameters that influence image contrast include sequence parameters, preparation pulses and contrast agents. 


\subsection{Contrast and contrast-to-noise}

The detection of tumors and other lesions in M R imaging depends on how well they are distinguished from the surrounding tissues. The image contrast is defined as the signal difference between two tissues. However, noise, either statistical or systematic, has tendency to mask the detection of lesions in MRI. In clinical practice the contrast-to-noise ratio is usually a more reliable measure of discerning lesions than contrast itself. The image noise has been considered the primary obstacle for detection of low-contrast lesions in M RI (Edelstein et al.1983). The statistical noise is more apparent at low field strengths. It can be reduced by increasing the voxel volume or the number of acquisitions per phase encoding, or by decreasing the sensitive volume of the receiver coil or the bandwidth. For maximizing CNR per unit time between two tissues of interest the optimal pulse sequences and sequence parameters should be carefully chosen by user.

\subsection{Spin echo imaging}

$M R$ imaging sequence describes the timing of RF pulses for spatial encoding and signal acquisition. In spin echo (SE) technique a slice selective excitation RF pulse is applied to generate transverse magnetization and simultaneously the longitudinal component of magnetization is reduced. The $180^{\circ}$ slice selective refocusing pulse inverts the accumulated dephasing, causing the appearance of an echo. This step is repeated with different phase-encoding steps as often as requested by the user. The signal intensity (S) for SE sequence can be approximated as follows (1):

$$
S \approx \rho f(v) e^{-T E / T 2}\left(1-e^{-T R / T 1}\right),
$$

where TR is the repetition time, TE the echo time, $\rho$ is the density of mobile spins in the object, and function $f(v)$ describes the effect of spins flowing with velocity $v$. It is assumed that TR $>$ TE. By choosing appropriate TR and TE values one can acquire images on which the contrast depends mainly on the differences of either $\mathrm{T} 1$ or $\mathrm{T} 2$ relaxation times.

\subsection{Gradient echo imaging}

The gradient echo (GRE) sequences have been assigned for fast image acquisition (Haase et al. 1986). The shorter imaging time of GRE imaging compared to SE imaging is a result of shorter TR values and smaller flip angles (FA). The refocusing $180^{\circ}$ pulse is not needed because reversal of the magnetic field gradients produces an echo. With a constant TR, the use of a large FA and short TE produces predominantly T1-weighted images and small FA and long TE predominantly T2-weighted images (Hendrick 1999). 


\subsubsection{GRE versus SE}

The transverse relaxation time, in the absence of RF refocusing pulse, is referred to as T2*. This includes not only the dephasing due to T2 spin-surroundings interactions, but also the additional dephasing mechanisms due to susceptibility gradients and magnetic field inhomogeneities. These two are compensated by $180^{\circ}$ pulse in SE sequences, but with gradient echo imaging all the dephasing mechanisms have to be considered. GRE technique requires a highly homogeneous static magnetic field in order to avoid image distortions and to achieve proper signal-to-noise ratio (SNR). In addition, the local field inhomogeneities arising from different magnetic susceptibilities of tissues within the body have a greater impact on GRE images than on SE images. These susceptibility gradients lead also to signal loss in the vicinity of tissue/air and tissue/bone interfaces. In head and neck imaging this can be observed at the skull base, and in sinonasal region, or in mouth region when metal objects are present. In conventional head and neck tumor imaging the SE techniques, which are less sensitive to susceptibility and motion artifacts are preferred over GRE techniques (Ross et al. 1994). On the other hand, the increased sensitivity of GRE to susceptibly gradients is favorable in detection of hemorrhagic lesions (Gomori et al. 1985).

\subsubsection{Chemical shift based opposed-phase imaging}

The resonance frequency spectrum of hydrogen bound to fat consists of several peaks, the largest of them being $3.5 \mathrm{ppm}$ (parts per million) lower than the resonance frequency of hydrogen bound to water. (Kamman et al. 1984). This frequency difference between protons in water and fat is called the chemical shift. It is directly proportional to $\mathrm{B}_{0}$ and leads to phase difference after $\mathrm{RF}$ excitation. In SE imaging this phenomenon is not a problem because $180^{\circ}$ refocusing pulse will rephase water and fat components at the time the echo is acquired. In GRE imaging the dephasing of fat and water components can lead to situation where the magnetization of protons in fat and water will point in the same or the opposite direction. These situations are TE dependent and they are called in-phase and opposed-phase, respectively. The signal intensity depends on fat and water content in a voxel, and phase difference between these molecules. The chemical shift based opposed-phase imaging has been used successfully for detection of fat in adrenal and ovarian tumor characterization (Bilbey et al. 1995, Honigschnabl et al. 2002, Imaoka et al. 1993) and for evaluation of vertebral metastasis (Uchida et al. 1993) and even for assessment of right ventricular dysplasia (Schick et al. 2000).

\subsection{The MRI preparation pulses to modify image contrast}

The RF pulses, which are used to modify image contrast before actual data sampling, are called preparation pulses. The preparation pulses are often non selective, which indicates that the excitation affects the whole imaging volume. The preparation pulses are generally used for suppression of signal from certain tissues to improve tissue contrast and lesion detectability. The two most frequently 
used suppression techniques include inversion recovery (IR) and chemical (spectral) saturation schemes. The magnetization transfer (Wolff and Balaban 1989) and spin lock (Sepponen et al. 1985) are other techniques that have been utilized for both enhanced tissue contrast and tissue characterization. Diffusion weighted imaging (DWI) has proved useful in the early detection of brain infarct (Sorensen et al. 1996) and tumor characterization (Holodny and Ollenschlager 2002)

\subsubsection{The inversion recovery techniques}

The inversion recovery (IR) techniques use $180^{\circ}$-inversion pulse prior to the actual imaging sequence to manipulate the contrast. After the inversion pulse the magnetization recovers exponentially through the null point. The time interval between the inversion pulse and the excitation pulse is inversion time ( $\mathrm{TI})$. The TI determines primarily the T1 weighting of the sequence and the T2-weighting is controlled by TE (Hendrick 1999). The IR technique can be used to improve T1 contrast, or to relaxation dependent suppression of tissues. Examples of latter are elimination of fat signal in short tau inversion recovery (STIR) imaging or elimination of cerebrospinal fluid in fluid attenuated inversion recovery (FLAIR) approach. In these techniques the excitation pulse of the imaging sequence is applied at the time the recovering longitudinal magnetization of fat or fluid is passing through the zero point, and consequently these tissues will not be excited. This zero point can be achieved by adjusting the TI to approximately 0.7 times of $\mathrm{T} 1$ relaxation time of tissue to be suppressed.

\subsubsection{Diffusion weighted imaging}

The diffusion technique involves the molecular motion of water protons, which in biological tissues is restricted by cell membranes or molecular boundaries. The extent of translational diffusion of molecules measured in the human body has been referred to as the apparent diffusion coefficient (ADC). Diffusion weighted imaging has been found sensitive for the early detection of acute brain infarct and for differentiation of acute from chronic infarct (Sorensen et al 1996). In addition, DWI has been evaluated in the characterization of different tumors in gynecology (Katayama et al. 2002), breast (Guo et al. 2002), liver (Namimoto et al. 1997) and especially in brain (Holodny and Ollenschlager 2002) with variable results. In one study (Sugahara et al. 1999) DWI was useful in the assessing of cellularity and grading of gliomas, whereas in another investigation (Lam et al. 2002) no distinction was possible between low grade and high-grade gliomas. Differentiation between dermoid/epidermoid and arachnoid cysts has been found reliable with DWI (Holodny and Ollenschlager 2002).

In a recent study (Wang et al. 2001) DWI and the determination of apparent diffusion coefficient were found promising in the characterization of head and neck tumors. The mean ADC of carcinomas was significantly smaller than that of solid benign tumors. In addition, the lymphomas and carcinomas could be differentiated by the means of ADC. With the ADC $1.22 \times 10^{-3} \mathrm{~mm}^{2} / \mathrm{s}$ as the threshold for predicting malignancy, the highest accuracy of $86 \%$ was obtained. 


\subsection{Head and neck MRI protocol for optimized detection of lesions}

It has been shown that fat-suppressed contrast enhanced T1-weighted fast spin echo (FSE) imaging provides the highest CNR for head and neck tumors and metastatic lymph nodes (Ross et al. 1994). In salivary gland tumor imaging the fat-suppressed T2 weighted FSE or inversion-recovery fast spin-echo sequences have given the highest lesion conspicuity (Sharafuddin et al. 1995). However, Takashima et al. (1997) found the T1 weighted nonenhanced images useful in detection of parotid masses. A routine protocol for head and neck neoplasms and associated adenopathy consists of T1 weighted and fat suppressed T2 weighted SE images, or preferentially FSE images (Lewin et al. 1994, Fulbright et al. 1994, Lenz et al. 2000). Additionally, T1 weighted fat suppressed images are applied with and without contrast medium to assess the vascularity of tumor (Lenz et al. 2000).

\section{Tissue characterization in conventional MRI}

\section{1 $\mathrm{T} 1$ and $\mathrm{T} 2$ relaxation times and tissue specificity}

The measurement of relaxation times has been one of the most commonly used approaches in M RI towards tissue characterization since Damadian's (1971) original observations on different tissues. Unfortunately, the determinations of T1 and $T 2$ relaxation times have been found unreliable in predicting tissue composition in later studies (Hollis et al. 1973, Inch et al. 1974, Dooms et al. 1985, Bottomley et al. 1987, Komiyama et al. 1987, Just and Thelen 1988, Lundbom et al. 1990, Wagner-Manslau et al. 1994). Most tumors and other pathological states such as inflammation are characterized by tissue edema and elevated T1 and T2 values, but the different pathological states do not differ significantly from each other (Bottomley 1987). The elevation of relaxation times in neoplastic tissues is usually less than that of resulting from inflammation, but there is a considerable overlap (Dooms et al. 1985, Wagner-M anslau et al. 1994).

The poor results in tissue characterization with relaxation time measurements may partly be due to technical problems related to the in vivo relaxation time measurements (Heaney and Ehman 1999). Physiological motion can produce random and systematic errors on images and calculated relaxation times. Technical circumstances of measurement such as the size and number of regions of interest (ROIs) and the partial filling phenomenon in small or heterogenic targets influence the relaxation time calculations. The number of data points to express exponential decay has to be large enough for obtaining reliable results. Additionally, the field strength has to be taken into account in relaxation time calculations. The multi-component T2 analysis might be more reliable in tissue characterization, but it is technically even more demanding. As a conclusion, determination of $\mathrm{T} 1$ and $\mathrm{T} 2$ relaxation times is a time consuming and challenging process, which has not been widely applied to clinical imaging. 


\subsection{Tissue characterization in head and neck tumor imaging}

Determination of $\mathrm{T} 1$ and $\mathrm{T} 2$ relaxation times has not been useful in characterization of head and neck tumors ( $M$ andelblatt et al. 1987). In conventional M R imaging, T2-weighted sequences have been reported to reflect underlying pathologic changes better than other pulse sequences and therefore to possess some potential for prediction of histologic nature of tumors (Som and Biller 1989, Sigal et al. 1992). On T2-weighted images, adenocystic carcinomas with low signal intensity corresponded to highly cellular tumors (solid subtype) with a poor prognosis; le sions with high signal intensity corresponded to less cellular tumors (cribriform or tubular subtype) with a better prognosis. However, MR images were not specific in differentiation of adenocystic carcinomas from other types of tumors ( $\mathrm{Si}$ gal et al. 1992). It has been suggested that highly malignant parotid tumors are characterized by unsharp margins (Vogl et al. 1990, Takashima et al. 2001) and low signal intensity on both T1- and T2-weighted images (Som and Biller 1989, Takashima et al. 1997). The authors suggested that in high-grade malignancies of parotid gland the cellularity and relative lack of free water and mobile protons produced a low T2-weighted signal. Low grade benign tumors with variable amounts of serous and mucous material, and inflammatory lesions with increased water content had higher T2 signal intensity (Som and Biller 1989). Takashima et al. (1997) found combination of hypointensity on non-fat suppressed T2-weighted FSE images and absence of hyperintensity on T1-weighted images to have $81 \%$ accuracy in predicting parotid gland malignancy.

Results contradictory to studies mentioned above have been reported in two studies with large number of patients by Freling et al. (1992) and Takashima et al. (1993). They found T2 hypointensity and evaluation of tumor margins unreliable diagnostic criteria in characterizing salivary gland tumors. Takashima et al. (1993) found hypointensity on T2-weighted images an unreliable diagnostic criterion in predicting malignancy in major salivary gland lesions. Only $18 \%$ of malignant tumors had hypointense signal on T2-weighted images and fibrosed benign tumors also showed hypointensity. In the study of Freling et al. (1992) no statistically significant correlation was found between parotid tumor signal intensity and histologic nature of the lesion.

\subsubsection{Lymph node imaging studies}

\subsubsection{Conventional methods}

Conventional $M R$, computed tomography $(C T)$ and sonography have all been shown to be superior to palpation in staging of head and neck cancer (Casteljins and van den Brekel 2001). The most sensitive method for staging NO neck is sonography-guided fine-needle aspiration cytology with reported sensitivity ranging from $48 \%$ to $73 \%$ and specificity $100 \%$ (Casteljins and van den Brekel 2001). The combined use of Doppler-blood flow pattern and size criteria has been demonstrated to increase the diagnostic accuracy (Yonetsu et al. 2001). $M$ easurements of $\mathrm{T} 1$ and $\mathrm{T} 2$ relaxation times of metastatic and normal lymph nodes have shown substantial overlap and failed to differentiate normal or in- 
flammatory nodes from metastatic nodes (Dooms et al. 1985, Wagner-M anslau et al. 1994). Wagner-M anslau et al. (1994) found $T 1$ relaxation time measurements useful in monitoring the tumor and metastatic lymph node response to radiochemothrapy. The hyperplastic lymph nodes showed significantly lower T1 values compared to malignant nodes, but the differentiation between inflammatory and malignant lymph nodes was not reliable. The commonly accepted criteria for metastatic head and neck node disease, which are based on size (van den Brekel et al. 1990), central necrosis or extracapsular spread, have been found to be of limited value in clinical imaging. Due to poor sensitivity for nodal micrometastases, the imaging modalities have seldom clinical consequences for the management of the head and neck cancer, and elective neck dissection is routinely performed.

\subsubsection{UItrasmall superparamagnetic iron oxide}

The most widely used paramagnetic contrast agent gadolinium diethylenetriamine penta-acetic acid (GD-DTPA) reduces T1 relaxation time and improves head and neck tumor detection (Lenz et al. 2000). Its effect correlates well with tissue vascularity and augments the assessment of tumor vascularity. Otherwise, Gd-DPTA does not provide much aid for tissue characterization, and the current focus in developing of new contrast agents is on increasing tissue specificity. UItrasmall superparamagnetic iron oxide (USPIO) is a new MR contrast agent, designed to the evaluation of reticuloendothelial system of lymph nodes (Anzai et al. 1997). Small iron oxide particles are taken up by macrophages within normally functioning nodes, reducing their signal on postcontrast M R because of the magnetic susceptibility effects of iron oxide. The signal of metastatic nodes, on the other hand, remains high on postcontrast T2*-weighted gradient echo images. Thus, the iron oxide-enhanced MR lymphography provides information about the anatomy, function and physiology of lymph nodes, and first clinical experiences in head and neck cancer patients suggested that it may improve the diagnostic accuracy for nodal metastases (Anzai et al. 1997).

Recently, a large multicenter clinical study was performed to evaluate USPIO enhanced M RI in diagnosis of lymph node metastases in patients with head and neck carcinoma (Sigal et al. 2001). The iron oxide-enhanced M R lymphography increased specificity compared to precontrast MR. However, precontrast MR showed an unexpectedly high sensitivity, which was not increased by administration of USPIO. False-positive results were partially due to inflammatory nodes, whereas false negative results related to the presence of undetected micrometastases. The authors concluded that the potential contribution of USPIO enhanced M R still remains limited due to technical problems regarding motion and susceptibility artifacts and spatial resolution. In the future these problems can be overcome and the role of USPIO is likely to gain importance in the clinical M R imaging of head and neck cancer. 


\section{Other imaging methods for enhanced tissue specificity}

The primary objective of head and neck tumor imaging has been to provide anatomic information to the staging of a tumor. The accuracy of conventional imaging methods M RI, CT and sonography in tumor characterization and especially in accurate detection of recurrent tumors or identification of cervical metastases has been disappointing. To resolve these problems the most active research has involved metabolic and functional imaging techniques (Mukherji et al. 2003).

\subsection{Positron emission tomography}

\subsubsection{Evaluation of recurrent tumors}

In conventional MRI the evaluation of post therapeutic tumor recurrence has proved to be unreliable (Chong and Fan 1997). In post therapeutic M RI high water content and hypercellularity of an immature scar cannot be differentiated from recurrent tumor because both show enhancement on T1-weghted images and have high signal intensity on T2-weighted images. A mature scar, which is mainly collagenous, hypocellular tissue, does not show enhancement and is characterized by low signal intensity on T2-weighted images.

Positron emission tomography (PET) is a functional imaging method that depicts tissue metabolic activity. The glycolytic activity has been shown to be increased in a variety of tumors. PET scanning with fluorine labeled deoxyglucose (FDG) appears promising in the evaluation of recurrent head and neck tumors (Chong and Fan 1997, M cGuirt et al. 1998, Greven et al. 2001). A malignant lesion has been demonstrated to accumulate significantly more FDG than post treatment fibrosis. PET scanning performed four months after radiotherapy has been shown to be superior in differentiating tumor recurrence from soft tissue irradiation effects in comparison to conventional imaging methods: computed tomography (CT) and M RI (M cGuirt et al. 1998, Greven et al. 2001).

\subsubsection{Unknown primary and nodal staging}

Variable but mostly promising results for FDG PET imaging have been demonstrated in the localization of the unknown primary site of origin in metastatic head and neck cancer (Aassar et al. 1999, Mukherji et al. 2003). In a prospective study by Adams et al. (1998) FDG PET imaging was shown to be superior to M RI and CT in the staging of head and neck cancer. The specificity of FDG PET imaging in detecting cervical metastases has been shown to range from $80 \%$ to $100 \%$. Since FDG is taken up nonspecifically by glycolytically active cells, it accumulates in the reactive and inflammatory lymph nodes as well as metastatic nodes, which is the major explanation for the false positives. In the evaluation of N 0 neck PET imaging is insensitive to the metastatic foci smaller than 3 to $4 \mathrm{~mm}$, but seems to detect smaller metastases than M R or CT imaging (Mukherji et al. 2003). The de- 
velopment of combined CT/PET scanners is expected to show higher accuracy for head and neck tumor imaging by combining the high anatomical detection of CT imaging with functional molecular information of PET scanning.

\subsection{Radioimmunoscintigraphy and molecular markers}

For the more accurate detection of nodal micro metastases or minimal residual disease diagnostic assays based on molecular markers have been developed (van Houten et al 2000). At present, there are two main methodologies in use, one based on antigen-antibody interaction and the other on amplified nucleic acids. The imaging technique related to the use of monoclonal antibodies is called radioimmunoscintigraphy (RIS). In a previous study by De Bree et al. (1995) RIS was evaluated in the staging of cervical nodal metastases with results similar to CT and MRI. In the immunohistochemical analyses monoclonal antibodies had targeted all tumor-involved lymph nodes, but the poor visualization by gamma camera imaging worsened the detection capability and sensitivity. Investigations to improve the accuracy of RIS are under way. One attractive idea is to integrate monoclonal antibodies into the modern PET imaging with CT/PET scanners (Mukherji et al. 2003).

\section{Magnetization transfer}

\subsection{The concept of magnetization transfer and MT effect}

In conventional MRI the acquired signal is emitted by hydrogen protons of freely moving water protons $(\mathrm{Hf})$ with relatively long $\mathrm{T} 2$ relaxation times. The protons associated with macromolecules or water molecules on the surface of macromolecules with restricted motion ( $\mathrm{Hr}$ ) have a broad line width, and a very short $\mathrm{T} 2$, causing their signal to decay too rapidly for detection in normal MRI (UImer et al. 1999). In magnetization transfer technique an off-resonance RF pulse with appropriate frequency is applied to saturate invisible $\mathrm{Hr}$ but not to affect $\mathrm{Hf}$. There is a constant exchange of magnetization between the two pools and the saturation of $\mathrm{Hr}$ is transferred to visible $\mathrm{Hf}$, which leads to lower signal in $\mathrm{MR}$ images. The contrast achieved with this technique is referred as magnetization transfer contrast (M TC) and it reflects the interaction between protons of freely moving water molecules and protons associated to macromolecules. Wolff and Balaban (1989) introduced an imaging method exploiting this M T contrast.

The observed decrease in signal intensity of the tissue with application of MT preparation pulse can be defined as a simple MT ratio (MTR) or MT effect (D ousset et al 1992).

$$
M T R=M T \text { effect }=\left[1-\left(M s / M_{0}\right)\right],
$$

where $\mathrm{M} 0$ is the magnetization of the free water pool without MT saturation and $M \mathrm{~s}$ is the magnetization with MT saturation. 
In the ideal case of complete $\mathrm{Hr}$ saturation, without any direct saturation of $\mathrm{Hf}$, the pseudo first-order rate constant $(\mathrm{K})$ of magnetization transfer is given by following equation (Balaban and Ceckler 1992).

$$
K=M T \text { effect/T1sat, }
$$

where T1sat is the T1 in the presence of MT irradiation. The determination of M T effect compared to determination of $K$ is a much less time-consuming process for clinical practice and has been widely used for tissue characterization (UImer et al. 1999). In a previous study both M T effect and K were found useful for characterization of brain tumors, but determination of $\mathrm{MT}$ rate was even more sensitive than M T effect (Kurki et al. 1996). Tumors had longer T1 and T1sat than normal white matter (Kurki et al. 1996), and therefore their reduced MT effects indicated an even more substantial reduction in their $\mathrm{MT}$ rate constant in relation to normal brain. It is good to notice that the MT effect does not equate to the rate of $M T$, and also that the $M T$ effect values are extremely technique dependent.

The MT effect is influenced by several factors: biological tissue properties, technical aspects of MT pulse, characteristics of the imaging sequence and the main magnetic field strength. The MT effect is elevated by increasing the duration or amplitude of the M T pulse, or by bringing the off-resonance frequency of the pulse closer to the water resonance (UImer et al. 1996). Previous works have shown that MT effect is dependent on the square root of the ratio of T1 to T2 (McGowan and Leigh 1994, Ulmer et al. 1996). Due to T1 dispersion the T1 values increase and the MT effects should be larger at high field strengths. This has been demonstrated by Duvvuri et al. (1999) who found M T effects of brain tissue to be more pronounced at $4 \mathrm{~T}$ compared to $1.5 \mathrm{~T}$. However, in the earlier studies with protein solutions it has been shown that the cross relaxation rates decrease with increasing field strength leading to increased T1 relaxation times (Zhong et al. 1989, Koenig et al. 1993). The presence of paramagnetic substances reduces the ratio of $\mathrm{T} 1$ to $\mathrm{T} 2$ and consequently also diminishes the observed M T effect (Kurki et al. 1992).

\subsection{MT in molecular level}

In studies of protein solutions it has been demonstrated that $\mathrm{MT}$ is sensitive to alterations in the molecular weight (Zhong et al. 1990), concentration (Iino 1994) and structure (Virta et al. 1995) of proteins. Immobilization of protein solutions by thermal or chemical denaturation (cross-linking) has been shown to increase magnetization transfer (Koenig et al. 1993, Virta et al. 1995). The nature of macromolecule surface water binding sites and the rotational correlation time of the macromolecule have been indicated to affect M T (Koenig et al. 1993).

It has been proposed that hydroxyl groups of cholesterol in myelin are the major locus for M T in white matter of the brain (Koenig et al. 1991, Fralix et al. 1991). Kucharczyk et al. (1994) found that many cerebrosides and phospholipides display prominent cross-relaxation as well. In Lundbom's study (1992) M T effects 
were correlated with high-molecular weight nuclear proteins in astrocytomas and with collagen content in meningeomas. In addition, collagen has been shown to possess an important source of MT in articular cartilage (Kim et al. 1993). Virta et al. (1996) demonstrated that the amount of nuclear material and chromatin contributed strongly to the measured magnetization transfer.

\subsection{Tissue characterization with MT}

MT contrast is determined by the interaction between protons of freely moving water molecules and protons associated to macromolecules. Thus, tissue macromolecules contribute significantly to the observed MT contrast. Determination of MT effects has been used on a variety of central nervous system lesions to enhance tissue characterization potential (Dousset et al. 1992, Lundbom 1992, M ittl et al. 1993, Mehta et al. 1993, Boorstein et al. 1994, Wong et al. 1995, Kurki et al. 1995, Kurki et al. 1996, Okumura et al. 1999). M T effect calculations have been proved useful for differentiating multiple sclerosis (MS) plaques from other white matter lesions (D ousset et al. 1992, Wong et al. 1995). The variation of M T effects in white matter and MS lesions has been suggested to reflect the extent of myelin loss (Dousset et al. 1992). Volumetric magnetization transfer imaging has been developed for the evaluation of myelination disorders in children (van Buchem et al. 2001). M T has been also employed for tissue characterization of liver tumors (O utwater et al. 1992, Halavaara et al. 1998), extraocular muscle disease (UImer et al. 1998) and for the evaluation of cartilage degeneration in knee (Koskinen et al. 1997).

\subsection{Tissue characterization of head and neck and brain tumors with MT}

Previous studies have demonstrated that MT technique has potential in characterization of brain tumors (Lundbom 1992, Kurki et al 1995, Kurki et al 1996, O kumura et al 1999). The M T effects of astrocytomas are shown to be lower than those of meningeomas and pituitary adenomas (Lundbom 1992). The M T effects of astrocytomas correlate with the degree of differentiation suggesting that the MT effects may reflect the amount of nuclear proteins known to be greater in high-grade astrocytomas. Kurki and co-workers (1996) demonstrated that MT parameters are superior to conventional parameters in brain tumor characterization and in the grading of gliomas.

In another previous investigation MT imaging was found useful in differentiating benign and malignant head and neck neoplasms (Yousem et al. 1994). H owever, MT effects were unable to distinguish different malignant tumors, and in contrast to Lundbom's (1992) work M T effects were also ineffective to differentiate the degree of cell differentiation within squamous cell carcinomas. More recent studies investigated the influence of the MT pulse frequency-offset and the effect of MTR determination method (lesion MTR vs. lesion-to-muscle MTR) for the prediction of malignancy in the head and neck (Takashima et al. 2000, Takashima et al. 2001). The lesion M TRs and the lesion-to-muscle M TRs in malignant tumors 
were statistically significantly greater than those in benign lesions at both 0.5 and $1.0 \mathrm{kHz}$ M T pulses, but no significant differences were noted between them at 0.3 $\mathrm{kHz}$ pulse. In this experimental setting lesion-to-muscle MTRs at a 1-kHz MT pulse frequency were shown to be optimal for diagnosis of head and neck tumors. With M TR of 0.61 or greater the highest accuracy of $85 \%$ was achieved. In a study of parotid masses a combination of margin characteristics and lesion-to-muscle magnetization transfer ratios was found useful in prediction of malignancy (Takashima et al. 2001).

\subsection{MT and contrast improvement}

Tanttu et al. (1992) and Kurki et al. (1992) were among first investigators to suggest that M T could have synergistic action with Gd-DTPA at $0.1 \mathrm{~T}$. The contrast of enhancing brain lesions can be improved by suppressing unenhancing surrounding tissues with $M T$ pulse and simultaneously reducing the signal suppression of the lesion with paramagnetic agent that shortens T1. The combined use of MT and Gd-DTPA has been shown to increase the contrast in brain lesion imaging in several studies (Tanttu et al. 1992, Kurki et al. 1992, Finelli et al. 1994, Mehta et al. 1995, Abo Ramadan et al. 1997). Finelli et al. (1994) and Mehta et al. (1995) demonstrated an approximate doubling of enhancing lesion CNR with MT for a variety of CNS lesions and CNR of the same order as triple dose of GdDTPA. In a study with low field strength $(0.1 \mathrm{~T})$ a significant but not as remarkable increase in brain tumor CNR was found with combined use of MT and GdDTPA (Abo Ramadan et al. 1997). Using the background suppression capability M T technique has been exploited in M R angiography (Edelman et al. 1992,) and also for contrast improvement in liver neoplasms (O utwater et al. 1992, Kahn et al. 1993, Halavaara et al. 1998), breast (Flamig et al. 1992), normal knee (Wolf et al. 1991) and heart (Balaban et al. 1991).

\section{6 $\mathrm{MT}$ and $\mathrm{T} 2$ relaxation}

The contrast in MT imaging is similar to T2-weighted imaging (Balaban and Ceckler 1992). It has been suggested that M T and T2 relaxation are parallel processes both of which are primarily governed by macromolecular structure and surface interactions between macromolecules and hydration water (UImer et al. 1999). H owever, it has been proposed that determination of M T effect may reveal tissue specific information not available on conventional T1-or T2-weighted images. Indeed, MT effect calculations have demonstrated differentiation between active edematous lesions and more chronic demyelinated lesions, despite similar appearances on T2-weighted images (Dousset et al. 1992, Tomiak et al. 1994). The MT effects of white matter have been shown to be lower in patients with multiple sclerosis (D ousset et al. 1992, Loevner et al. 1995) or brain metastases (Boorstein et al. 1994) in spite of normal appearing white matter with conventional imaging techniques. H owever, these studies compared conventional images that were eval- 
uated qualitatively with quantitatively analyzed MT effects. A recent study with more sophisticated analyzing methods demonstrated the discrimination potential of calculated T2 values and showed significantly longer T2 values of normal-appearing white matter in MS patients than in healthy subjects (Whittall et al. 2002). A comparative quantitative study in tissue characterization between MT parameters and $\mathrm{T} 2$ determinations has not yet been performed.

\section{Spin lock imaging}

\subsection{SL technique}

With spin lock method, first introduced by Jones (1966) and applied for imaging by Sepponen et al. (1985) and Santyr (1989) it is possible to obtain information on low field relaxation processes with a high signal to noise ratio. In the on resonance SL technique, the magnetization is tilted into the transverse plane and locked with the use of a very low radio frequency locking field (B1L) applied on resonance to the water peak (Sepponen et al. 1985). The proton relaxation takes place along the locking field and it is not influenced by the much higher main magnetic field. The relaxation process is analogous to the T1 relaxation and characterized by the relaxation time T1p. The advantage of SL technique is that the image contrast is characterized by the relaxation processes which are effective at low field strengths, while the high signal-to-noise is maintained by the main magnetic field. Energy absorption is a limitation with SL imaging. The specific absorption rate $(S A R)$ is proportional to the square of the product $B_{0}{ }^{*} B L$, where $B L$ is the RF field or RF preparation pulse amplitude (Sepponen 1992). Human on-resonance SL imaging is limited because of possible heating of tissues to the low field M RI strengths. The off-resonance SL technique has been developed for SL imaging at high field strengths to overcome the energy absorption problem (Santyr et al. 1989). In offresonance $\mathrm{SL}$ experiment the RF pulse is applied off-resonance to the water peak but closer to the resonance than in MT technique. The relaxation occurs along the effective relaxation field $B_{\text {eff }}$ with relaxation time $T 1 \rho_{\text {off }}$

It has been demonstrated that $T 1 \rho$ and $T 1$ values are close to each other at frequencies between $0.01-0.1 \mathrm{M} \mathrm{hz}$ (Santyr et al. 1989). Thus, the favourable tissue contrast and tissue discrimination potential combined with low field T1 imaging (Koenig et al. 1984) may also be achieved with T1 $\rho$ technique at all B0 fields. However, if the B1L is smaller than the local fields of the nuclei those spins are not locked and the $T 1 \rho$ is close to $T 2$ measured at $B_{0}$ (Sepponen et al 1985). It has been shown in protein solutions that $T 1 \rho$ at $B 1 L=0$ is equal to $T 2$ measured at $\mathrm{B}_{0}=0.1 \mathrm{~T}$ (Virta et al. 1997). The contrast on SL images resembles T2 weighted images, especially at low locking field strengths (Sepponen et al.1985, Mulkern et al. 1989, Ulmer et al. 1996, Engelhardt and Johnson 1996, Virta et al. 1998a). 


\subsection{Evaluation of contrast with SL imaging}

The contrast in SL imaging is generated during the locking pulse without application of gradients, and the dephasing of spins is reduced compared to T2 weighted imaging. Thus, SL imaging is less sensitive to diffusion losses caused by susceptibility artifacts as demonstrated by Engelhardt and Johnsson (1996). They found $\mathrm{T} 1 \rho$ as an alternative contrast parameter to $\mathrm{T} 2$ in magnetic resonance histology at 9.4 T, with fewer susceptibility artifacts. Ulmer et al. (1996) studied the effect of varying saturation pulse frequency-offsets on image contrast in neuroimaging at 1.5 T. The near-resonance SL preparation pulse produced T2-like contrast to all other imaging sequences. The increased myocardium-blood contrast with and without contrast medium in SL-GRE images was found at 1.5T (Dixon et al. 1996, Semple et al. 1998). In a recent study spin-lock flow tagging was employed successfully for M R angiography (Azhari et al. 2001). In liver tumor imaging multiple slice SL technique has produced image contrast comparable to magnetization transfer technique (Halavaara 1998). Santyr and co-workers (1989, 1994) showed improved tumor detection for T1 $\rho$ imaging at $0.15 \mathrm{~T}$ and $1.5 \mathrm{~T}$ compared to conventional T1 and T2 parameters in breast tissue. SL imaging contrast properties have been evaluated also for brain (Mulkern et al. 1989, Aronen et al. 1999) and muscle imaging (Virta et al. 1998a, Virta et al. 1998b).

\subsection{SL imaging and tissue characterization}

It has been demonstrated in protein solutions that $1 / T 1 \rho$ is sensitive to the alterations in the molecular weight, concentration and structure of proteins (Virta et al. 1997). The SL effect has been shown to be useful in differentiating hemangiomas from liver metastases (Halavaara et al. 1995, Halavaara et al. 1998). Santyr et al. (1989) reported T1 $\rho$ to be more effective in differentiating tumors from normal breast tissue when compared to T1 or T2 relaxation times. SL imaging has been found useful for evaluating cartilage degeneration (Koskinen et al. 1997, Duvvuri et al. 1997, M lynarik et al. 1999, A kella et al. 2001). The T1 $\rho$ values were longer in advanced cartilage degeneration compared to intermediate cartilage degeneration at 0.1 T (Koskinen et al. 1997). In their studies, Duvvuri et al. (1997) and Akella et al. (2001) showed T1 $\rho$ measurements of patellar cartilage to be selectively sensitive to proteoglycan content and to reveal structural abnormalities not found on conventional T1- or T2-weighted images.

In recent studies, Gröhn et al. (2000) and Kettunen et al. (2001) showed that T1p measurements were sensitive for early changes of cerebral ischemia in rat at 4.7 T. The T1 $\rho$ increased within minutes after onset of ischemia. A much slower change in MT contrast was evident over the first 20 min of ischemia. It has been suggested that immediate increase in T1 $\rho$ may be caused by changes in microscopic susceptibility and tissue acidosis rather than by net water gain into the cell. Later, in the consolidating infarction the extensive prolongation of T1 $\rho$ and the decrease of MT are associated with water accumulation into tissue (Kettunen et 
al. 2002, M äkelä et al. 2002). The tissue T1 $\rho$ regulation by pH is mediated possibly through its effect on proton exchange. Hakumäki et al. (2002) evaluated Ganciclovir treatment response in gliomas. $T 1 \rho$ relaxation time proved to be more sensitive indicator of cytotoxic cell damage and of early Ganciclovir treatment response than conventional M RI contrast parameters.

\subsection{Comparison between SL and MT imaging}

In MT technique the RF off-resonance preparation pulse (saturation pulse) is applied selectively to saturate the bound protons of tissue while leaving the mobile tissue water protons unaffected. In off-resonance SL experiment the preparation pulse is employed closer to the resonance than MT pulse with variable direct saturation effect of mobile water protons depending on frequency-offset and amplitude of the preparation pulse (UImer et al. 1996). In on resonance SL technique the preparation pulse is applied at Larmor frequency saturating directly the mobile water protons. In MT technique a very low amplitude preparation pulses can be applied. In SL technique the amplitude of the locking pulse must be higher than the local fields of tissue nuclei; otherwise the locking of the spins is released by the thermal energy of molecules (Sepponen et al. 1985).

The degree of direct saturation for an MT or SL experiment can be determined by calculating suppression effects of a manganese chloride $(\mathrm{MnCl})$ phantom (Ulmet et al. 1996). The more the $\mathrm{MnCl}$ is suppressed the more the direct saturation (or spin locking effect) exists. Ulmer et al. (1996) suggested with their pulse design, an offset frequency of $300 \mathrm{~Hz}$ to be optimal for clinical neuroimaging. The net effect of reducing the frequency offset from 2000 to $300 \mathrm{~Hz}$ increased T2 contrast in T1, T2 and proton density images.

Tissues with short or intermediate $\mathrm{T} 1$ and a relatively large $\mathrm{T} 1 / \mathrm{T} 2$ ratio were susceptible to spin locking (UImer et al. 1996). Thus, the SL technique probes all relaxation mechanisms effective at very low field strengths. These include the paramagnetic relaxitivity of $\mathrm{MnCl}$ molecules and also thermal vibration of fat molecules. The MT technique is primarily sensitive only to macromolecule water interaction in tissues. However, it has been proposed that in protein rich tissues MT is the major relaxation mechanism also in T1p relaxation (Brown and Koenig 1992).

\subsection{T1 $1 \rho$ dispersion imaging}

By varying the strength of the locking field, the $T 1 \rho$ relaxation time changes and a variable tissue contrast can be produced to images. This field dependence of T1 $\rho$

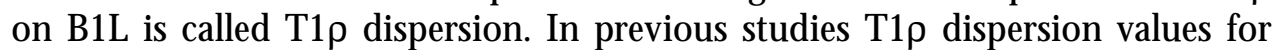
normal tissues at low field strengths have been measured (Tanttu et al. 1986, Virta et al. 1997a, Koskinen et al. 1999). Abdominal organs with high macromolecular protein content like the liver, pancreas and muscles demonstrated a considerable dispersion in a low B1L range of $50 \mu \mathrm{T}-500 \mu \mathrm{T}$. Fat and water rich tissues had a negligible T1 $\rho$ dispersion. 
Previously, a few MRI studies have been made with the T1 $\rho$ dispersion technique to evaluate human diseases (Sepponen et al. 1986, Santyr et al. 1989, Sepponen et al. 1990, Lamminen et al. 1993, Virta et al 1998b). In their study, Lamminen and co-workers (1993) observed significantly lower T1 $\rho$ dispersion values in muscle tissue of patients with dystrophic or myopathic disease compared to muscle tissue of healthy volunteers. However, no statistically significant difference was found be tween the muscle dispersion values of patients with different myopathies. In the study of multiple sclerosis (Sepponen et al. 1990) the plaques had longer T1 $\rho$ and a slightly smaller T1 $\rho$ dispersion than normal brain tissue (B1L: $50 \mu \mathrm{T}$ to $200 \mu \mathrm{T}$ ).

T 1 $p$ dispersion imaging has also been studied in brain infarcts at 0.02 T (Sepponen et al. 1986) and more recently at $4.7 \mathrm{~T}$ in rats (Gröhn et al. 2000). Sepponen and associates (1986) demonstrated that fresh brain infarctions had longer $\mathrm{T} 1 \rho$ and smaller T1 $\rho$ dispersion compared to normal brain tissue (B1L: $50 \mu \mathrm{T}$ to $460 \mu \mathrm{T}$ ). This was proposed to reflect the higher water content and slightly decreased number of macromolecules in a fresh infarction. Gröhn and co-workers (2000) showed that T1 $1 \rho$ dispersion imaging detects an irreversible cerebral ischemia earlier than conventional MRI methods. T1 $\rho$ dispersion increased by approximately $20 \%$, comparable with the magnitude of diffusion reduction. In a recent study (Tailor et al. 2003) T1 $\rho$ dispersion imaging was successfully applied in the quantitation of cerebral blood flow in rats at $4 \mathrm{~T}$. The authors recommended the low-frequency SL technique as safe and suitable also for human perfusion imaging applications at high fields.

\section{Low field versus high field}

\subsection{The field dependence of relaxation and contrast}

Based on the various relaxation time studies it has been proposed that the low field range provides specific information related to water/macromolecular interaction because more relaxation processes are effective at that range and they are assumed to reflect the low frequency components associated with the macromolecules (Bocard 1984, Conti 1984). Generally, T1 relaxation times of tissues become shorter towards low fields leading to the greater variability of T1 values at low field than at high field strength (Koenig et al. 1984). The variability of T1 values at low fields has been shown to exceed the variation of tissue water content. This seems to give a potential for better tissue discrimination at low fields. T2 relaxation time has much weaker field strength dependence. H owever, the susceptibility effect is larger at high field strength which results in somewhat shorter T2* relaxation times. Theoretically, the signal-to-noise is proportional to the square of $B_{0}$ which results in a diminished SNR at low fields. However, the better inherent tissue contrast at low field strengths reduces the loss in CNR. Thus, the CNR, which characterizes diagnostic capability of MRI image, has weaker $B_{0}$ dependence than SNR (Sepponen 96).

It has been suggested that in protein rich tissues magnetization tranffer is the major source of relaxation at very low magnetic field strengths $\left(B_{0}\right)$ (Zhong et al. 1989, Zhong et al. 1990, Koenig et al. 1993), and magnetization transfer is also a 
dominant relaxation mechanism in $\mathrm{T} 1 \rho$ relaxation observed by spin lock imaging (Brown and Koenig 1992). This is also supported by the observation that T1 of muscle tissue at very low field strengths $\left(B_{0}\right)$ is approximately $T 1 \rho$ at locking field conditions (B1L) (Santyr et al. 1989).

\subsection{Clinical imaging and field strength}

$M R$ units with $B_{0}$ below $0.3 T$ have been considered low-field scanners and units with $B_{0}$ over $1 T$ are considered high-field scanners (Sepponen 1996). Considering safety aspects there are a few advantages in low field imaging compared to high fields. The magnetic field produces magnetomechanical forces on ferromagnetic objects, which possess a potential health risk in M RI. The magnetomechanical force is dependent on $\mathrm{B}_{0}$ and involves a smaller risk at low field strength. For the patient convenience, the acoustic noise caused by gradient operations is weaker at low field strength.

The RF absorption of biological tissues is described by specific absorption ratio which is proportional to the square of the product $B_{0}{ }^{*} B L$ (Sepponen 1992). Because the electric losses in the body increase as the frequency increases the RF power needed for excitation or preparation pulses also increases at high field strengths and may more easily cause local heating of tissues. At low field strength the RF irradiation power needed is lower and utilization of preparation pulses is more convenient. This applies to on resonance SL technique where exceeding of SAR limits may be problematic. However, the development of SL techniques with off-resonance irradiation and short locking pulse lengths (TL) has resolved this problem and made SL technique also available at high field strengths (Sepponen 1996).

The difference between precessional frequencies of hydrogen protons in fat and in water is proportional to the field strength, resulting in minor chemical shift artifacts at low field strengths compared to high fields. The adverse effect of smaller chemical shift is that the chemical or frequency-selective presaturation of fat is more demanding at low field strength. However, a good absolute homogeneity of $\mathrm{B}_{0}$ is easier to achieve at low field than at high field strength which augments spectral saturation of fat at low fields. Motion and susceptibility artifacts are minor problems at low field strengths and the GRE sequences which are more sensitive to these artifacts than SE sequences can be more freely utilized at low fields. 


\section{AIMSOF THE STUDY}

The purpose of the study was to explore and develop imaging sequences with tissue characterization potential for head and neck tumor imaging at $0.1 \mathrm{~T}$. The specific aims were:

To determine $T 1 \rho$ relaxation times for head and neck tissues and tumors at $0.1 \mathrm{~T}$, and to compare the values with respective $T 1$ and $T 2$ relaxation times (III, IV).

Based on these relaxation time parameters to explore and develop multiple slice spin lock gradient echo (SL-GRE) sequences for head and neck tumor imaging (IV).

To compare the tumor contrast on multiple slice SL out of phase fat/water images with spin echo (SE) T2 weighted images in head and neck tumor imaging (IV).

To evaluate and compare the SL, Tlp dispersion and MT imaging techniques in the differentiation of benign and malignant head and neck tumors $(\mathrm{I}, \mathrm{II})$. 


\section{MATERIALS AND METHODS}

\section{Patients and normal volunteers}

Fifty three patients with histologically $(n=49)$ or cytologically $(n=4)$ confirmed head and neck tumors were studied (I, II, IV). Of these, 40 patients were included in study I, 24 in study II and 40 in study IV. There were 17 patients who participated in both studies I and II. Ten healthy volunteers between the ages 25 and 45 years were imaged in study III. Study IV consisted of two parts. For the evaluation of imaging parameters the study group consisted of six healthy volunteers and six patients. For the comparison of SL- and T2- weighted sequences 34 patients with head and neck tumors were imaged. Number of patients with salivary gland tumors was 14,9 and 11 in studies I, II, and IV, respectively.

\section{MR imaging protocols}

M R imaging was performed on $0.1 \mathrm{~T}$ clinical M R device (M erit, M arconi M edical Systems Finland Ltd. Vantaa, Finland). Either a multipurpose neck coil or a head coil was used.

In studies I and II conventional T2-weighted SE (1500/30/120 ms) and multiple slice GRE (1500/30 ms) SL and M T images were obtained. Based on these multiple slice images an appropriate slice that showed well the pathology was chosen for further evaluation. SL, MT and T1 $\rho$ dispersion single-slice interleaved images (two interleaved images for each sequence, one with a weaker and the other with a stronger preparation pulse) were obtained with GRE sequence (1500/30 ms).

T1p dispersion single-section images (II, III) were obtained with two different amplitudes of B1L: $35 \mu \mathrm{T}$ and $300 \mu \mathrm{T}$. The locking pulse length $(\mathrm{TL})$ was set to $100 \mathrm{~ms}$. The locking field parameters were selected because T1 $\rho$ dispersion is known to be effective in this range of amplitudes (Knispel et al. 1974, Tanttu et al. 1986).

\subsection{Single slice SL sequence}

The saturation pulses in the MT sequence had the amplitudes of $0 \mu \mathrm{T}$ and $13 \mu \mathrm{T}$ with duration of $500 \mathrm{~ms}$ (Toff). The offset from the resonance frequency of water was $4 \mathrm{kHz}$.

The SL sequence used for calculations of SL effect $(I, I I)$ is described in Fig. 1. The spin lock pulse with amplitude BIL of $35 \mu \mathrm{T}$ was applied before a GRE sequence. Two different locking pulse lengths TL $10 \mathrm{~ms}$ and $100 \mathrm{~ms}$ were used (TLa, $T L b)$. Because the sequence controller of the imaging device did not allow us to use TL of $0 \mathrm{~ms}$, the shortest possible TL of $10 \mathrm{~ms}$ was chosen. Two images corre sponding to each pulse length were reconstructed. In the rising part of the pulse, 
the RF frequency-offset is swept from $2.5 \mathrm{kHz}$ to $0 \mathrm{~Hz}$. This tilts the magnetization as an adiabatic sweep from the $z$-axis to the xy-plane. The magnetization is then locked in the direction of the RF field for a time of TLa or TLb. In the falling part the frequency-offset sweeps back to $2.5 \mathrm{kHz}$ leaving the magnetization in the direction of the z-axis. As a result, the magnetization is prepared by an amount depending on T1 $\rho$ and TL. Immediately after the locking pulse follows the excitation pulse and the signal is read as a GRE.

\subsection{Multiple slice SL sequence}

The multiple slice SL-GRE (1500/30) sequence (I, II, IV) had following imaging parameters: B1L $35 \mu \mathrm{T}, \mathrm{TL} 10 \mathrm{~ms}$ and $\alpha 60^{\circ}$. In the multiple slice SL imaging technique, non-selective locking pulses are applied between the successive excitations of slices for generation of $\mathrm{T} 1 \rho$-weighted contrast in the whole imaging volume (Sepponen et al. 1993, M oran and Hamilton 1995; Fig. 2). The effect of successive locking pulses is cumulative. When the repetition time of the locking pulses (TD) is shorter than T1 of tissues the generation of contrast assumes only short locking pulse lengths. Because the contrast is generated during the locking pulses, TE may be selected to optimize signal-to-noise ratio or improve the contrast between tissues. By keeping TL and TE short a large number of slices $(\mathrm{N})$ within a given TR

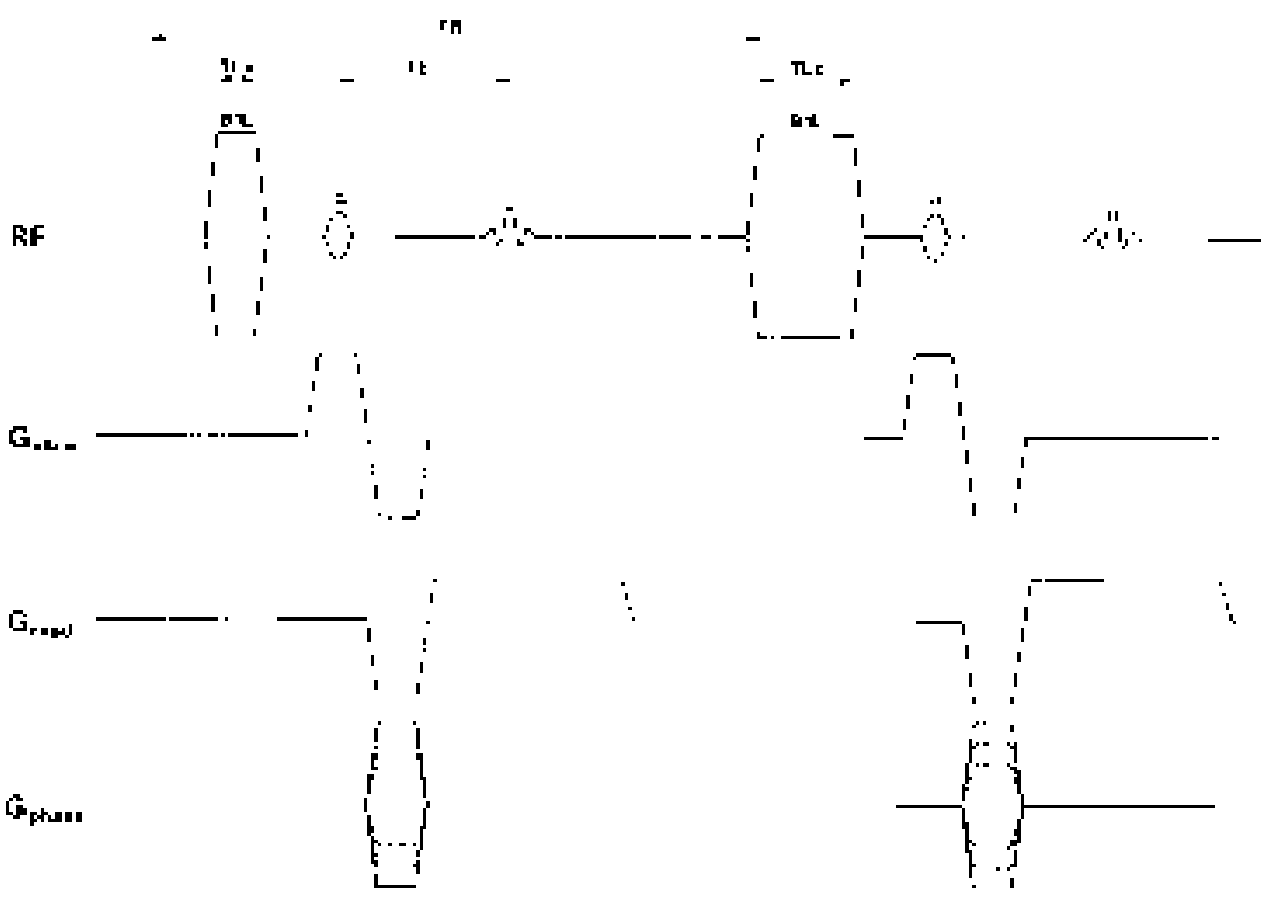

Figure 1. Diagram of the spin lock sequence used to measure SL effect. The locking pulses with amplitude BIL and lengths of TLa and TLb were used in an interleaved manner. The echo was acquired as a gradient echo. 
can be acquired. The intensity of signal S from muliple slice $S L$ sequence is given approximately by Equation 4 (Tanttu 1999):

$$
S=S_{0} \sin (a) * E 2 * \frac{1-(E D * E L)^{\wedge} N}{1-(E D * E L)^{\wedge} N * \cos (\alpha)} * \frac{(1-E D) * E L}{1-E D * E L},
$$

where $E 2=e^{-T E / T 2}, E D=e^{-T D / T 1}, E L=e^{-T L / T 1 \rho}$,

$\alpha$ is the flip angle, $N$ is the number of slices, and TD is the time period between successive locking pulses.

\section{Determination of SL, MT and T1p dispersion effects (I, II, III)}

Regions of interest (ROIs) within the tumors were placed on the area with the largest reduction in signal intensity, as determined by the application of weaker and stronger SL or MT pulses. The size and shape of ROIs were kept constant. The SL effect and the MT effect were calculated as 1 - (intensity with stronger preparation pulse/intensity with weaker preparation pulse). T1 1 dispersion effect was defined as 1 - (signal intensity with lower locking field amplitude/signal intensity with higher locking field amplitude).

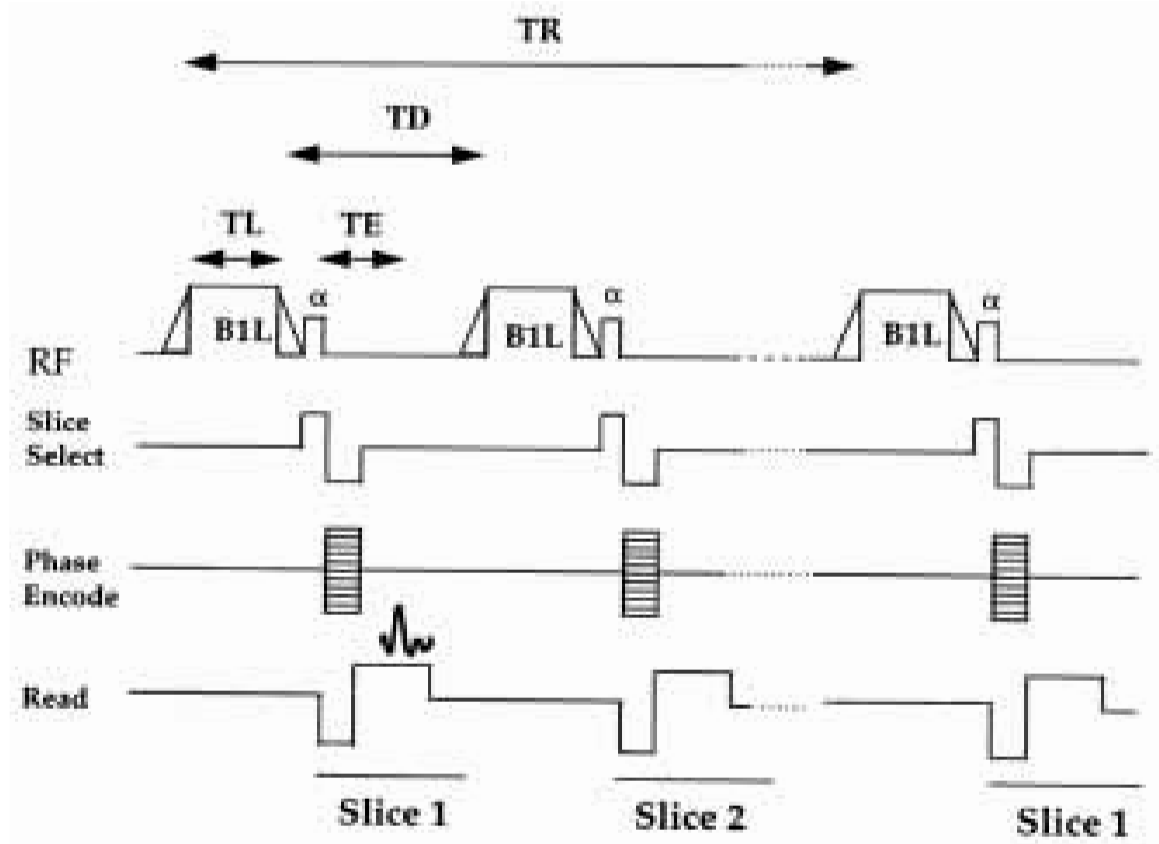

Figure 2. The multiple slice spin lock sequence. $T R$, repetition time; TD, time delay between successive locking pulses; $T L$, pulse length; $T E$, echo time; $R F$, radiofrequency; $B 1 L$, locking pulse amplitude; $\alpha$, flip angle. 


\section{Relaxation time measurements (III, IV)}

\subsection{T1 and T2 measurements}

The T1s of tissues were obtained by using a multiple slice inversion recovery sequence ( $T R=1500 \mathrm{~ms}, T E=30 \mathrm{~ms}$ (III), TE $=40 \mathrm{~ms}$ (IV) with three different inversion times TI $=50 \mathrm{~ms}, 414 \mathrm{~ms}$, and $814 \mathrm{~ms}$. In study III GRE and in study IV SE sequence was used. The T2 values were determined with a SE (1500/40/120 $\mathrm{ms}$ ) sequence. T1 and T2 values were calculated by fitting the measured tissue signal intensities to the corresponding proton relaxation equations with the leastsquares method.

\subsection{T1p measurements}

The $T 1 \rho$ relaxation times for different tissues and tumors were determined by using a single-slice SL-GRE (1500/30 ms) sequence in study III and a single slice SLSE (1500/40 ms) sequence in study IV. Four interleaved transaxial images with TLs of $10 \mathrm{~ms}, 30 \mathrm{~ms}, 60 \mathrm{~ms}$, and $140 \mathrm{~ms}$ were obtained. The B1L was $35 \mu \mathrm{T}$. To calculate T $1 \rho$, the measured signal intensities were fitted to Equation 5 (Sepponen et al. 1985).

The intensity of signal (S) from single slice SL-sequence can be approximated as follows (Eq. 5):

$$
\mathrm{S}=\mathrm{S}_{0}\left(1-\mathrm{e}^{-\mathrm{TR} / \mathrm{T} 1}\right) \mathrm{e}^{-\mathrm{TL} / \mathrm{T} 1 \rho} \mathrm{e}^{-\mathrm{TE} / \mathrm{T} 2}
$$

where $S_{0}$ is the maximum signal intensity produced by the equilibrium magnetization at the polarizing magnetic field.

\section{Exploring multiple slice SL imaging parameters (IV)}

By using the relaxation time information, approximate equations were used to evaluate theoretical contrast-to-noise ratios for pairs of tumor and different head and neck tissues. The epidermoid carcinoma with T1 $\rho$ of 112 ms was chosen as a representative tumor in these CNR and relaxation time parameter evaluations.

The CN R between tumor and tissue was calculated using equation 6 .

$$
\mathrm{CNR}=\left(\mathrm{S}_{\text {tumor }}-\mathrm{S}_{\text {tissue }}\right) /\left(\mathrm{S}_{\text {tissue }}+\text { noise }\right),
$$

The TR was set long enough ( $T R=1500 \mathrm{~ms}$ ) to reduce the $T 1$ effect on contrast. B1L was $35 \mu \mathrm{T}$ and slice thickness was $5 \mathrm{~mm}$. The effect of imaging parameters $\mathrm{TL}, \alpha, \mathrm{N}$ and TE on image CNR was evaluated. 


\section{Comparison of tumor CNR between SL and T2-weighted sequences (IV)}

Conventional axial SE T2-weighted (1500/30/120 ms) and multiple slice axial SLGRE (1500/30 ms) images were obtained. Based on the theoretical analysis as described above, the following parameters were chosen for the multiple slice SL sequence: B1L $35 \mu \mathrm{T}$, TL $10 \mathrm{~ms}$ and $\alpha 60^{\circ}$. The TE $=30 \mathrm{~ms}$ for SL-GRE sequence was chosen to acquire opposed phase fat/water images.

The total imaging times and number of slices acquired with SE sequence were 4 minutes 48 seconds and 9 slices ( 28 patients) and 9 minutes 36 seconds and 18 slices ( 6 patients); and with SL sequence 6 minutes 24 seconds and 14 slices. As a consequence, the net imaging time/slice for SE technique was 32 seconds and for SL technique 27 seconds. The slice thickness was $7 \mathrm{~mm}$.

The signal intensity values were obtained from the ROIs placed on the tumor area and on the adjacent tissue. If there were two or more types of adjacent tissues, the tissue which had longest borderline with tumor was chosen (muscle $n=$ 14 , salivary gland $n=13$, fat $n=2$, sinus mucosa $n=4$, sinus air $n=1$ ). Additionally, we calculated and compared CNRs between tumor and adjacent salivary gland tissue $(n=16)$, between tumor and adjacent muscle $(n=16)$ and between tumor and fat $(n=34)$. The CNR for all salivary gland tumors $(n=11)$ was also determined. The CNR values were calculated using equation (6) as described earlier.

\section{Pathologic evaluation}

For the histologic examinations tissue samples were formalin fixed and paraffin embedded. Three to five $\mathrm{mm}$ thick sections were cut and stained with Hematoxylin-eosin (HE), van Gieson (vG) and periodic-acid-Schiff (PAS). Diagnostic immunohistochemistry was performed when needed. For cytologic examinations cell material was fixed in $50 \%$ alcohol, cytocentrifuged and stained according to Papanicolaou. A pathologist experienced in head and neck diseases retrospectively reviewed the slides of all the cases without any knowledge of the imaging data.

\section{Statistical methods}

In study 1 , the data analysis was performed either with the unpaired one-tailed $t$ test or the $M$ ann-Whitney $U$ test. The analysis by means of Student $t$ test assumes a normal distribution of two sets of data points. In our data the distribution of benign tumors had two peaks (not normally distributed), which makes the analysis with the $M$ ann-Whitney $U$ test more suitable. For non salivary gland tumors a $\mathrm{t}$ test was performed. Parametric simple linear regression analysis (Pearson) was used to assess whether there was any correlation between the SL and MT effects in tumors. 
In study II, data analysis was performed with the M ann-Whitney $U$ test. The sample size in benign and malignant tumor groups was not large enough to reliably assess normal distribution, which made the analysis with the nonparametric $M$ ann-Whitney $U$ test more suitable. The whole tumor data (benign and malignant together) and normal tissue data assumed better a normal distribution and parametric simple linear regression analysis (Pearson) was used to assess whether there was any correlation between the T1 $\rho$ dispersion, SL or M T effects in tumors or normal tissues.

In studies III, IV, the statistical data analysis was performed by using a paired $t$ test to compare the relaxation times between different tissues and to compare the tumor CNR values between T2-weighted images and SL images. Parametric simple linear regression analysis (Pearson) was used to assess the correlation between tumor CNR values on T2-weighted SE images and on multiple slice SL-GRE images.

In all studies values of $p<0.05$ were considered statistically significant. 


\section{RESULTS}

\section{Head and neck tissue characterization (I, II, III)}

\section{$1.1 \mathrm{SL}$ and MT effects of tumors (I, II)}

Of the 40 patients 20 had malignant and 20 benign tumors including five infections. The different tumors and their SL and MT effect values are listed in Table 1, and graphically illustrated in Fig. 3. Both SL and MT effects were higher for malignant (Fig. $2 / I)$ than benign tumors (Fig. $3 / I)(0.52 \pm 0.08$ vs. $0.42 \pm 0.15, p=0.09$ and $0.36 \pm 0.10$ vs. $0.25 \pm 0.13, p=0.02$, respectively, Mann-Whitney $U$ test). When infections were excluded the difference between malignant and benign tumors became statistically significant both with SL effect $(p=0.02)$ and MT effect

Table 1. SL and MT effects of different tumors

\begin{tabular}{lcccc}
\hline Tumor type & $\begin{array}{c}\text { No. of tumors } \\
\text { (SL) }\end{array}$ & SL effect* & $\begin{array}{c}\text { No. of tumors } \\
\text { (MT) }\end{array}$ & M T effect* \\
\hline Malignant & $\mathbf{2 0}$ & $\mathbf{0 . 5 2 \pm 0 . 0 9}$ & $\mathbf{1 8}$ & $\mathbf{0 . 3 6 \pm 0 . 1 0}$ \\
Epidermoid carcinoma & 9 & $0.53 \pm 0.01$ & 9 & $0.40 \pm 0.04$ \\
Epidermoid carcinoma & & & & \\
(necrotic) & 1 & 0.20 & 1 & 0.01 \\
Lymphoma & 5 & $0.55 \pm 0.05$ & 4 & $0.37 \pm 0.03$ \\
Acinic cell carcinoma & 1 & 0.58 & 1 & 0.33 \\
Adenocystic carcinoma & 1 & 0.50 & 1 & 0.34 \\
Melanoma & 1 & 0.49 & 0 & \\
Neuroblastoma & 1 & 0.54 & 1 & 0.37 \\
Hypernefroma metastasis & 1 & 0.49 & 1 & 0.36 \\
\hline Benign & $\mathbf{2 0}$ & $\mathbf{0 . 4 2 \pm 0 . 1 5}$ & $\mathbf{1 7}$ & $\mathbf{0 . 2 5 \pm 0 . 1 3}$ \\
Pleomorphic adenoma & 5 & $0.37 \pm 0.13$ & 5 & $0.26 \pm 0.12$ \\
Basal cell adenoma & 1 & 0.57 & 1 & 0.42 \\
Adenolymphoma & 1 & 0.56 & 1 & 0.37 \\
Acinocellular tumor & 1 & 0.38 & 1 & 0.27 \\
Paraganglioma & 2 & 0.38 & 1 & 0.20 \\
Angiofibroma & 1 & 0.39 & 1 & 0.17 \\
Lipoma & 1 & 0.43 & 1 & 0.01 \\
Cyst & 2 & 0.19 & 2 & 0.13 \\
Mucocele & 1 & 0.59 & 1 & 0.34 \\
Infection & 5 & $0.50 \pm 0.17$ & 3 & $0.29 \pm 0.18$ \\
\hline Values & & & & \\
\hline
\end{tabular}

*Values are mean \pm standard deviation 


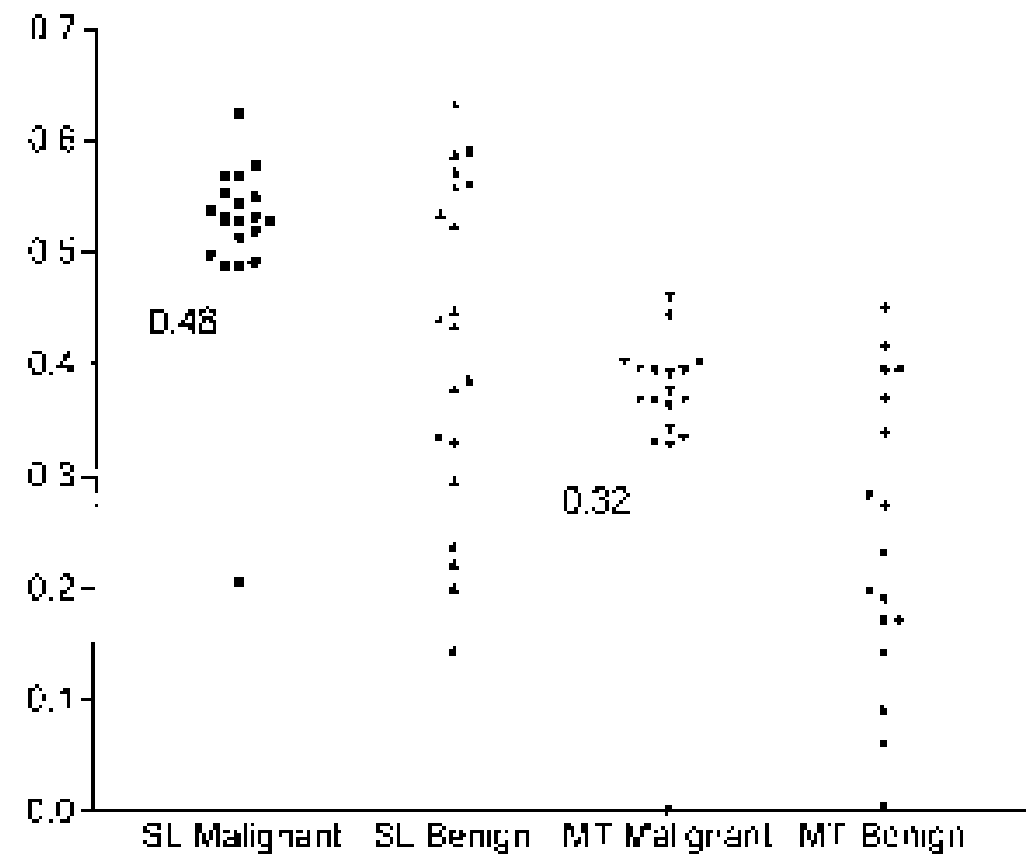

Figure 3. Calculated SL $(n=40)$ and M T $(n=35)$ effects in malignant (mean SL effect, $0.52 \pm$ 0.08 ; mean M T effect, $0.36 \pm 0.10$ ) and benign (mean SL effect, $0.42 \pm 0.15$; mean M T effect, $0.25 \pm 0.13$ ) tumors. The cutoff values for SL and MT effects are 0.48 and 0.32 , respectively.

( $p=0.007)$. When major salivary gland tumors were excluded ( 14 were studied with SL imaging, 13 with MT imaging) the differences in SL and MT effect were statistically significant ( $p=0.03, p=0.003$, respectively).

With an SL effect of 0.48 and an M T effect of 0.32 as the threshold, the sensitivity for detecting a malignant tumor was $95 \%$ and $94 \%$, specificity $60 \%$ and $65 \%$, and accuracy $78 \%$ and $80 \%$ for SL effect and MT effect, respectively. All the malignant tumors except one (an epidermoid carcinoma) had an SL effect of more than 0.48 and an M T effect of more than 0.32 . On the other hand, four benign tumors had SL and M T effects over these thresholds (pleomorphic adenoma, basal cell adenoma, adenolymphoma, and mucocele). Additionally, four infections had an SL effect of more than 0.48 , and two infections had an MT effect more than 0.32 (in two of the cases with SL effects of more than 0.48 , MT was not performed).

Comparison of the measured SL and MT effects in head and neck tumors showed strong correlation $(r=0.85, p<0.001)$ between the two modalities (Fig. 4). The only exception was a lipoma that showed no MT effect $(0.01)$ whereas its SL effect was 0.43 .

If the seven patients of study two, who were not included in study one, were added to the patient population ( $n=47)$, the differences in SL and M T effects be tween benign and malignant head and neck tumors became statistically signifi- 


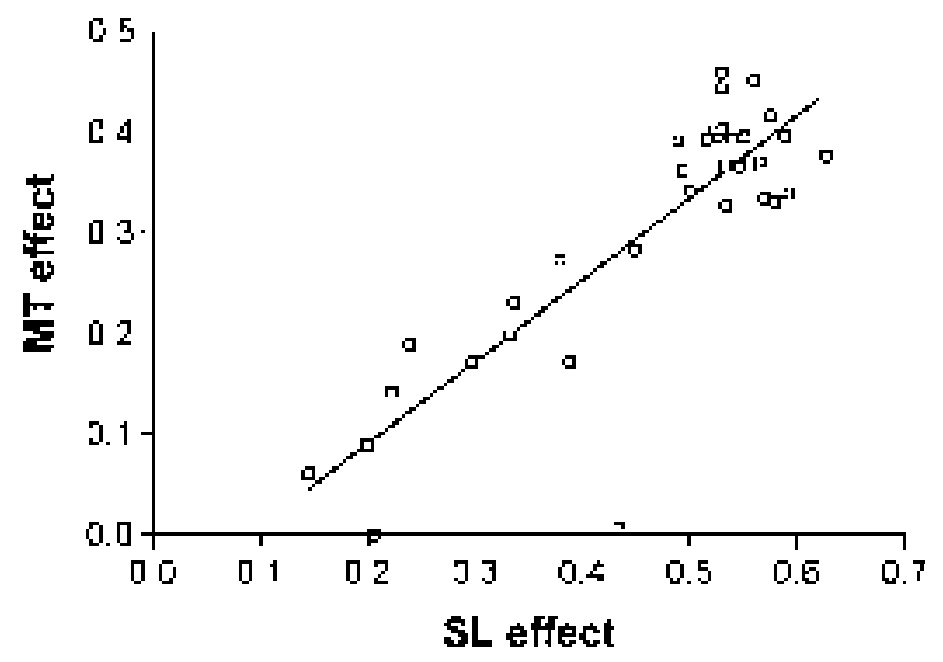

Figure 4. Correlation between SL and MT effects in different tumors ( $n=35, r=0.85, P<$ 0.001).

cant with both techniques $(0.53 \pm 0.08$ vs. $0.41 \pm 0.15, p=0.006$ and $0.36 \pm 0.09$ vs. $0.24 \pm 0.12, p=0.003$, respectively, $M$ ann-Whitney $U$ test). With an SL effect of 0.48 and an M T effect of 0.32 as the threshold, the sensitivity for detecting a malignant tumor was $96 \%$ and $90 \%$, specificity $67 \%$ and $71 \%$, and accuracy $81 \%$ and $81 \%$ for SL effect and MT effect, respectively.

\subsection{T2 evaluation of tumors (I, II)}

The T2-weighted signal intensity characteristics were also evaluated by comparing the signal intensity of tumors to the signal intensity of parotid gland tissue. When hypointensity of tumor on T2-weighted images was considered as a criterion for malignancy, the sensitivity was $20 \%$, specificity $90 \%$ and accuracy $60 \%$ in study I. In study II sensitivity was $60 \%$, specificity $92 \%$ and accuracy $77 \%$.

\subsection{SL and MT effects of normal tissues (I)}

The SL and MT effects of tumors and normal tissues are listed in Table 2. No substantial differences were noted between the SL and MT effects of normal glandular tissues and malignancies. However, there was a statistically significant difference between the $S L$ and MT effects of glandular tissues and benign lesions $(p=0.001, p<0.001$, respectively). There was also a statistically significant difference $(p<0.001)$ between the SL and MT effects of muscles and both malignant and benign tumors. Fat showed no MT effect $(-0.01 \pm 0.05)$, whereas the SL effect was $0.42 \pm 0.03$. 
Table 2. SL and MT effects of tumors and normal tissue

\begin{tabular}{lcccc}
\hline Measured tissue & No. (SL) & SL effect* & No. (MT) & MT effect* \\
\hline Malignant tumors & 20 & $0.52 \pm 0.08$ & 18 & $0.36 \pm 0.10$ \\
Benign tumors & 20 & $0.42 \pm 0.15$ & 17 & $0.25 \pm 0.13$ \\
Muscle & 40 & $0.70 \pm 0.07$ & 35 & $0.61 \pm 0.06$ \\
Salivary gland & 19 & $0.54 \pm 0.07$ & 14 & $0.39 \pm 0.09$ \\
Fat & 40 & $0.42 \pm 0.03$ & 35 & $-0.01 \pm 0.05$ \\
Reference(M MCl) & 40 & $0.33 \pm 0.02$ & 35 & $0.03 \pm 0.03$ \\
\hline
\end{tabular}

$*$ Values are mean \pm standard deviation

\section{$1.4 \mathrm{~T} 1 p$ dispersion imaging of tumors (II)}

In study II, of the 24 patients, 11 had malignant tumors and 13 had benign tumors including two infections. The different tumors and their T1 $\rho$ dispersion effect values are listed in Table 1/II. The T1p dispersion, SL and MT effects of different tumors are also graphically illustrated in Fig. 4/II.

T1 $\rho$ dispersion effects were higher for malignant than benign tumors. There was a statistically significant difference between malignant and benign tumors ( $0.20 \pm 0.05$ vs. $0.08 \pm 0.08, p=0.001$, Mann-Whitney $U$ test). T1 $\rho$ dispersion effects in head and neck tumors showed a strong correlation with SL and MT effects ( $r=0.87, p<0.001$ and $r=0.90, p<0.001$, respectively).

All the malignant tumors except a plasmocytoma had a Tl $\rho$ dispersion effect of more than 0.14 and MT effect of more than 0.32 (Fig. 4/II, Fig. 7/II). All malignant tumors had an SL effect of over 0.48 . With a T $1 \rho$ dispersion effect of 0.14 as the threshold, the sensitivity for detecting a malignant tumor was $91 \%$, specificity $77 \%$ and accuracy $83 \%$. When an SL effect of 0.48 and an MT effect of 0.32 were used as the cut-off values, the sensitivities were $100 \%$ and $89 \%$, the specificities were $77 \%$ and $82 \%$ and the accuracies were $88 \%$ and $85 \%$, respectively.

\subsection{T1p dispersion effects of normal tissues (II, III)}

The T1 $\rho$ dispersion, SL and M T effects of tumors and normal tissues are presented in Table 2/II. The most powerful T1 $\rho$ dispersion effect was achieved in muscle tissue $0.3 \pm 0.10$. There was a statistically significant difference between the $T 1 \rho$ dispersion effects of muscle and both benign and malignant tumors $(p<0.001$, $\mathrm{p}<0.005$, respectively, $\mathrm{Mann}$-Whitney $\mathrm{U}$ test). Fat and $\mathrm{MnCl}$ reference showed negligible T $1 \rho$ dispersion effect and MT effect, whereas their SL effects were moderate. The T1 $1 \rho$ dispersion effects for muscle, fat and $\mathrm{MnCl}$ were close to each other in both studies (II, III).

In study II, parotid gland tissue showed T1 $\rho$ dispersion effect of $0.10 \pm 0.12$. The variability (standard deviation/mean) for parotid gland tissue effects was 
large and there were six overlapping values between parotid gland and malignancies; on the other hand three parotid glands had negative effects. The T1 $\rho$ dispersion effects of parotid gland tissue were lower and variability greater in study II compared to study III $(0.10 \pm 0.12$ vs. $0.18 \pm 0.09)$. Congruently, the MT effects were lower and the variability greater in study II as compared with study I $(0.29 \pm 0.19$ vs. $0.39 \pm 0.09)$. SL effects of salivary gland tissues were close to each other in both studies (I, II). In study II there were three parotid glands with negligible T1 $\rho$ dispersion and MT effects and moderate SL effects, probably due to fat degeneration in glandular tissue.

\section{The relaxation time measurements of different tissues and tumors (III, IV)}

The T $1 \rho$ relaxation times measured at B1L of $35 \mu \mathrm{T}$ with SL-SE technique and T1 and $\mathrm{T} 2$ relaxation times of different head and neck tissues and tumors are presented in Table 3. The T1 $p$ values were slightly longer than T2 values for muscle, tongue, lymphatic and parotid gland tissues. The T1 $\rho$ relaxation time of fat tissue was considerably longer than $\mathrm{T} 2$ relaxation time and slightly shorter than T1 value.

The T $1 \rho$ and T1 relaxation times were determined in study III by GRE technique and in study IV by SE technique. T1p relaxation times in milliseconds ( $\mathrm{ms}$ ) of tongue and parotid gland were considerably shorter in study III as compared with study IV ( $43 \pm 6 \mathrm{~ms}$ vs. $65 \pm 8 \mathrm{~ms}$ and. $84 \pm 10 \mathrm{~ms}$ vs. $115 \pm 9 \mathrm{~ms}$, respectively). $\mathrm{T} 1$ relaxation times of tongue and parotid gland were considerably longer in study III compared to study IV ( $334 \pm 33$ ms vs. $276 \pm 25 \mathrm{~ms}$ and. $376 \pm 56 \mathrm{~ms}$ vs. $277 \pm 47 \mathrm{~ms}$, respectively). T1 of fat was $131 \pm 11 \mathrm{~ms}$ in study III and $164 \pm 20 \mathrm{~ms}$ in study IV. All the other relaxation times were close to each other between studies III and IV.

\section{Evaluation of multiple slice SL imaging parameters (IV)}

The evaluation of imaging parameters was based on relaxation time values obtained with SE technique and presented in Table 3. Generally, we observed that when number of slices was increased shorter TL values were needed to obtain optimal SL contrast (Fig. 5). By reducing $N$ and using longer TLs, the tumor CNR could be slightly increased (Fig. 5).

For the multiple slice SL (1500/30) sequence (14 slices) the CNR values be tween malignant tumor and tongue, or between malignant tumor and muscle, or between malignant tumor and lymphoid tissue were highest with $T L$ values be tween $10-35 \mathrm{~ms}$ and flip angles between $60^{\circ}$ and $90^{\circ}$ (Figs. 5 and 6). A relatively long TE was necessary to obtain high CNR. With TE of $40 \mathrm{~ms}$, the optimum TL is in the range of 10 to $20 \mathrm{~ms}$ (Fig. 4/IV). 
Table 3. T1p, T2, and T1 relaxation times of tumors and different tissues

\begin{tabular}{lllll}
\hline M easured Tissue & & $\mathrm{T} 1 \rho^{*}(\mathrm{~ms})$ & $\mathrm{T} 2^{*}(\mathrm{~ms})$ & $\mathrm{T} 1^{*}(\mathrm{~ms})$ \\
\hline Muscle & $(\mathrm{n}=11)$ & $51 \pm 11$ & $43 \pm 5$ & $272 \pm 17$ \\
Tongue & $(\mathrm{n}=5)$ & $65 \pm 8$ & $45 \pm 9$ & $276 \pm 25$ \\
Parotid gland & $(\mathrm{n}=11)$ & $115 \pm 9$ & $77 \pm 8$ & $277 \pm 47$ \\
Lymphatic tissue & $(\mathrm{n}=7)$ & $98 \pm 12$ & $72 \pm 6$ & $355 \pm 49$ \\
Fat & $(\mathrm{n}=11)$ & $142 \pm 9$ & $89 \pm 4$ & $164 \pm 20$ \\
Sinus retention & & 349 & 217 & 690 \\
Epidermoid ca & $(\mathrm{n}=2)$ & $112 \pm 6$ & $91 \pm 1$ & $440 \pm 46$ \\
Lymphoma & & 122 & 87 & 437 \\
Esthesioneuroblastoma & & 142 & 109 & 520 \\
Carcinoma ductale & & 91 & 63 & 380 \\
Pleomorphic adenoma & & 296 & 220 & 967 \\
\hline
\end{tabular}

${ }^{*}$ Values represent mean \pm standard deviation

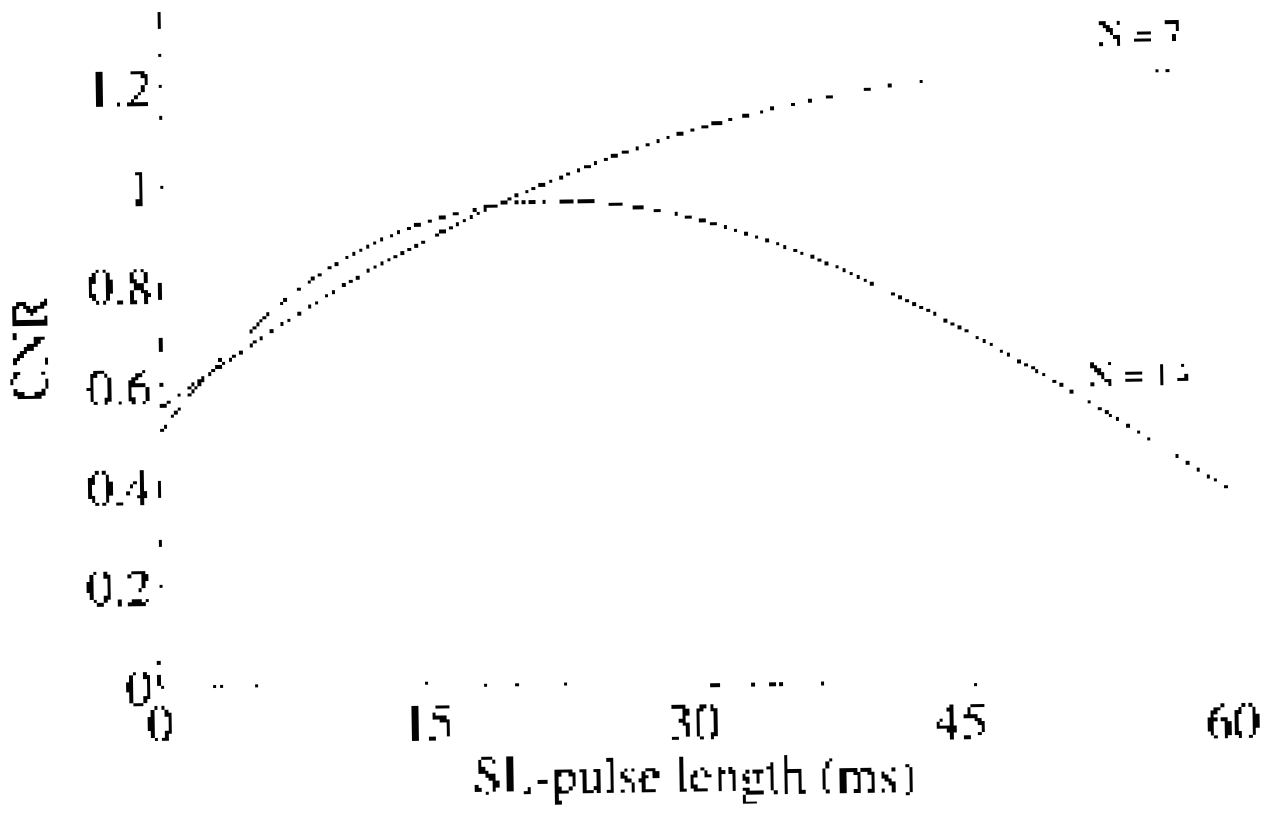

Figure 5. Contrast-to-noise ratio (CNR) between epidermoid carcinoma and muscle for multiple slice spin lock (SL; 1500/30) sequence (flip angle $90^{\circ}$ ) as a function of the SL-pulse length and the number of slices ( $N=7, N=14)$. By reducing the number of slices, CNR can be increased, but longer locking pulse lengths are required to optimize CNR. 


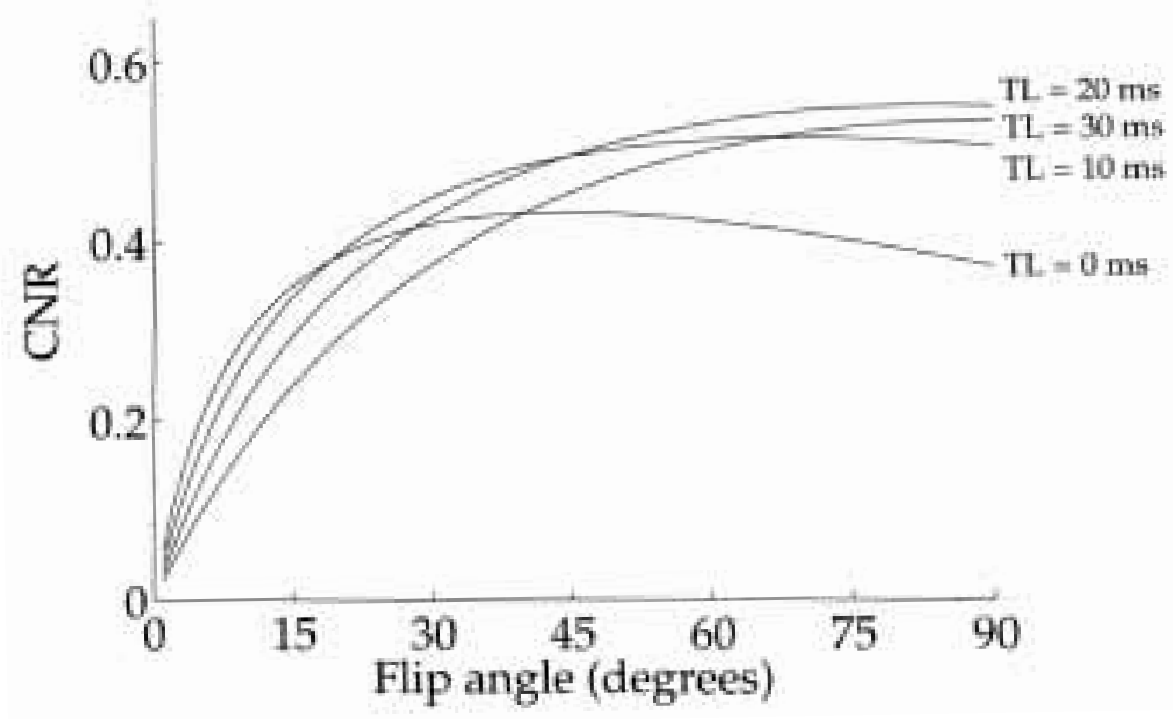

Figure 6. Optimization of Contrast-to-noise ratio (CNR) between epidermoid carcinoma and tongue for multiple slice SL (1500/30) sequence $(N=14)$ as a function of flip angle. The highest CNR values are obtained with locking pulse length (TL) of $20 \mathrm{~ms}$ and with flip angles in the range of $60^{\circ}$ to $90^{\circ}$.
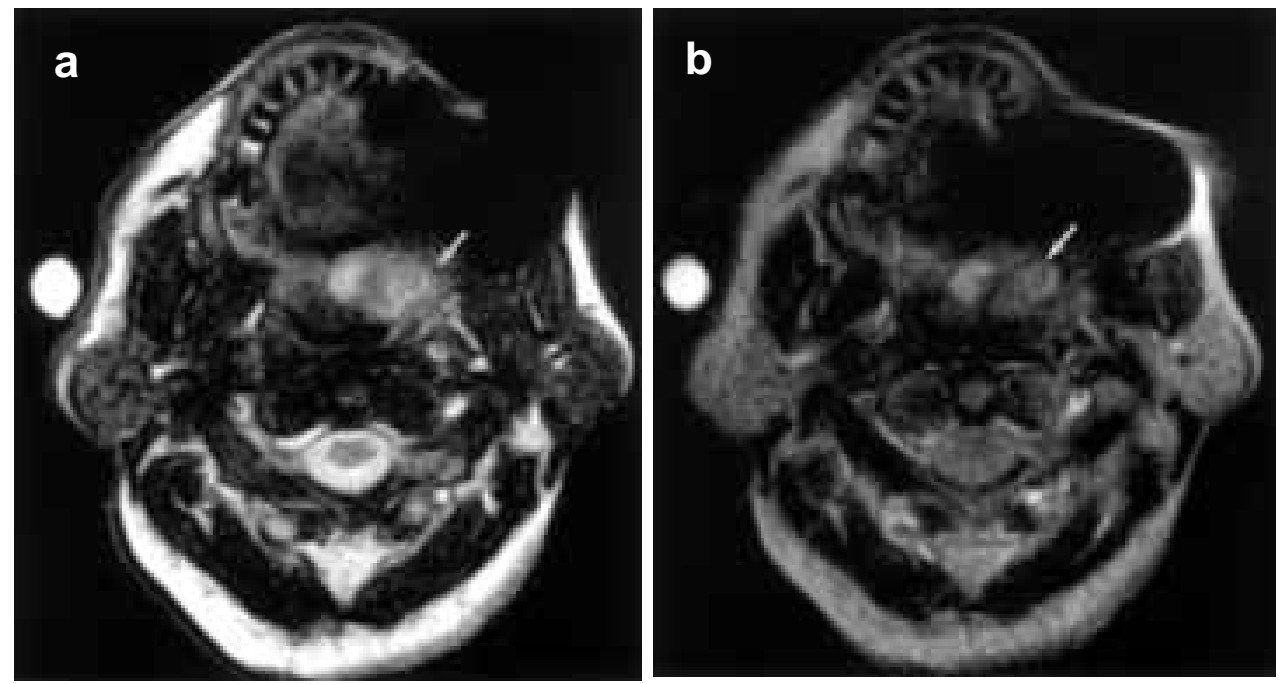

Figure 7. (a) Multiple slice spin lock gradient echo (1500/30) image and (b) T2-weighted spin echo image (1500/120) of left nasopharyngeal adenocystic carcinoma infiltrating the soft palate (arrow) to show the effect of susceptibility artifact on image quality. The metal object in the left molar region causes artifacts to both images. The image quality and contrast are better with the spin lock gradient echo technique, probably due to the short echo time (30 ms vs. 120 ms in the T2-weighted image). 


\section{Comparison of tumor CNR between SL and T2-weighted se- quences (IV)}

Of the 34 patients 17 had malignant (Figs. 7 and 8) and 17 benign tumors (Fig. 9). The mean CNR for tumors was similar on SL images compared to SE images ( $1.1 \pm 0.8$ vs. $1.0 \pm 0.8, p=0.5$, respectively, $t$ test). We also found a good correlation $(r=0.64, p<0.001)$ in tumor CN Rs between SL images and T2-weighted SE images.
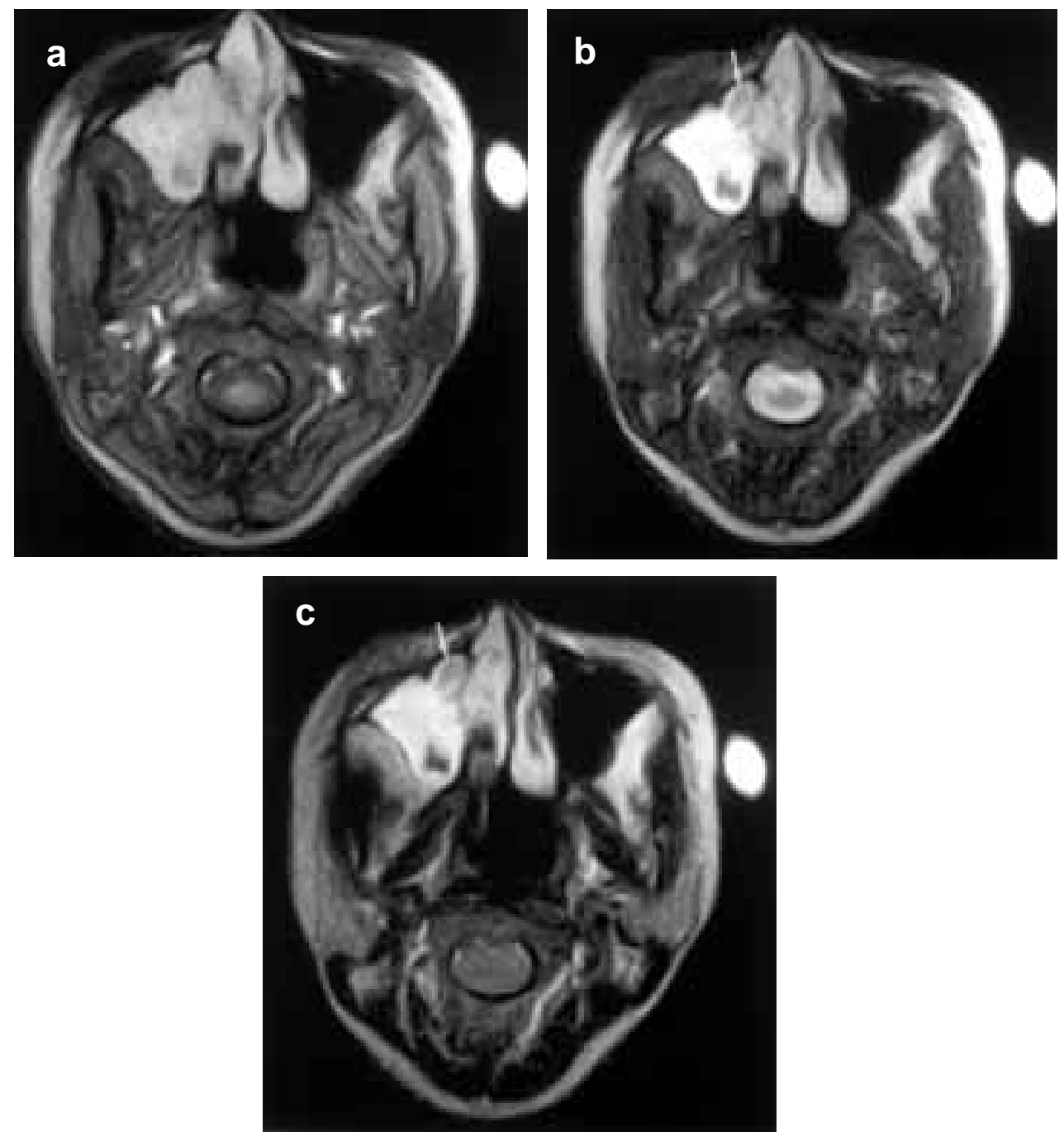

Figure 8. (a) Proton density gradient echo image (1500/30), (b), spin lock gradient echo image (1500/30) and (c) T2-weighted spin echo image (1500/120) of ethmoid esthesioneuroblastoma. Adding a spin lock pulse to a proton density sequence without changing the echo time leads to easier detection of a hypointense tumor (arrow in b and c) from inflammatory maxillary sinus retention on spin lock images compared with proton density images. The conspicuity of tumor is the same on spin lock and T2-weighted images. 
Those tumors adjacent to muscle had higher CNR values on T2-weighted SE images than on SL images $(p=0.02, n=14)$.

For salivary gland tumors ( $n=11,10$ benign and one malignant tumor) the mean CNR was significantly greater with the SL technique than with the T2weighted SE technique ( $1.2 \pm 0.6$ vs. $0.8 \pm 0.6, p=0.03$, respectively, $t$ test). When all of the tumors adjacent to salivary gland tissue ( $n=16,11$ benign and 5 malignant) were taken into account the difference between these techniques in tumor versus glandular tissue CN Rs remained significant $(p=0.03)$.

There were two parotid gland tumors (basal cell adenoma and infection); they were isointense on T2-weighted SE images, with very poor conspicuity. Three of the tumors adjacent to salivary gland were hypointense and 11 were hyperintense on T2-weighted images. With the SL imaging all tumors adjacent to salivary gland were hyperintense and easy to detect.
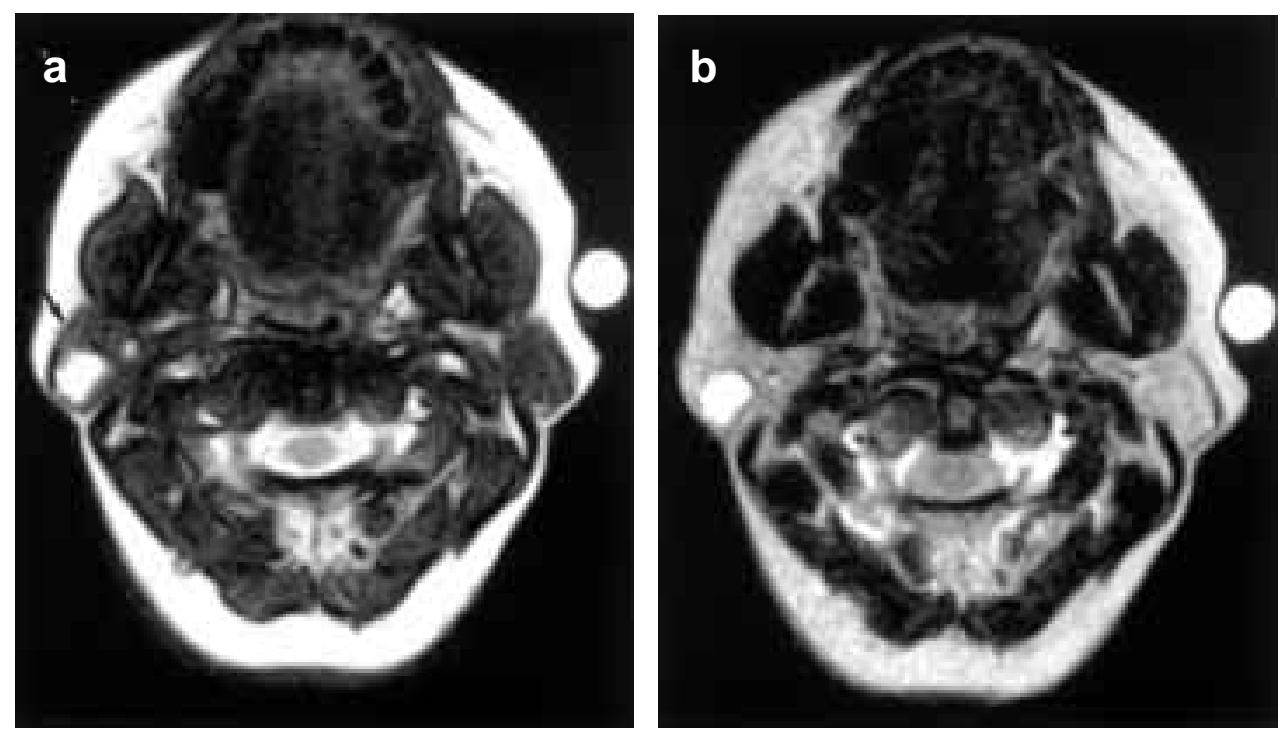

Figure 9. (a) Spin lock gradient echo image (1500/30) and (b) T2-weighted spin echo image $(1500 / 120)$ of right parotid pleomorphic adenoma. The overall contrast is similar with both techniques, but the parotid gland tissue (arrow in a) is more suppressed on the spin lock image, providing better tumor contrast. 


\section{DISCUSSION}

\section{Determination of relaxation parameters for head and neck tissues (III, IV)}

Our relaxation time calculations for muscle and fat are consistent with those obtained in previous studies at $0.1 \mathrm{~T}$ (Virta et al. 1998a, Koskinen et al. 1999). Earlier studies have demonstrated that on resonance SL technique produces T2-like image contrast (Sepponen et al. 1985, Mulkern et al. 1989, Ulmer et al. 1996, Engelhardt and Johnson 1996, Virta et al. 1998a). Our results with T1 $\rho$ values close to $\mathrm{T} 2$ relaxation times were in accordance with these previous findings. However, fat tissue demonstrated considerably longer T1p than T2. The high signal appearance of fat on T2-weighted FSE images is well known and has been attributed to reduced diffusion-mediated susceptibility dephasing and reduced Jcoupling (Williamsson et al. 1996). The signal intensity of fat increases as the repetition frequency of the refocusing $\pi$-pulses increases. At high repetition frequency the FSE technique approaches the SL experiment and probably the maintenance of in-phase condition during the locking pulse explains also the long T1p observed with the SL technique.

The T 1 and T1p relaxation times of different head and neck tissues were determined with GRE technique in study III and with SE technique in study IV. Fat containing tissues such as tongue and parotid gland demonstrated different relaxation times between these two studies. The explanation could be that determination of relaxation times for fat containing tissues with GRE technique is dependent on which TE times are used. Therefore, for fat containing tissues, the relaxation time parameters obtained with GRE technique in study III are strictly applicable only for contrast evaluation and optimization of the M RI sequence for opposed phase imaging with TE $30 \mathrm{~ms}$. For general evaluation of MRI contrast, the determination of relaxation times with SE technique is more convenient, because the influence of TE can be ignored. This is the main reason that in study IV we determined the relaxation times with SE technique.

\section{Multiple slice SL technique (IV)}

\subsection{Evaluation of imaging parameters}

In the multiple slice SL imaging when the number of slices is large the signal intensity and contrast of tissues with high macromolecular content have been shown to depend approximately on the ratio $\mathrm{N} * T L / T R$. The higher the ratio, the more T1 $\rho$ weighting there is on the resulting images. (Sepponen et al. 1993). Our results were consistent with this as we demonstrated that when the number of slices increases, shorter TL values are required to obtain optimal SL contrast (Fig. 
5). The highest tumor CNR values for multiple slice ( $N=14)$ SL-GRE (1500/30) imaging were achieved with locking pulse lengths between 10 and $35 \mathrm{~ms}$ and flip angles from $60^{\circ}$ to $90^{\circ}$ (Figs. 5 and 6). However, increasing $\alpha$ over $30^{\circ}$ had only small effect on CNR.

TR of $1500 \mathrm{~ms}$ was chosen for the patient study to reduce T1 effects and to achieve reasonable number of slices. TE of $30 \mathrm{~ms}$ was chosen to reduce signal bandwidth and to achieve opposed phase fat/water images. With TL of $10 \mathrm{~ms}$, adequate contrast was produced and more slices could be obtained with SL technique than with T2-weighted SE imaging.

\subsection{Evaluation of contrast}

In addition to T1p the contrast in multiple slice SL imaging is al so based on $T 1$ and T2 relaxations. As discussed above, T1 $\rho$ and T2 contrasts are synergistic in SL imaging, whereas T1-weighting has an antagonistic effect on T1p contrast. The shorter the tissue T1 is in comparison to time delay between successive locking pulses, the less T $1 \rho$ contrast will be generated. Thus, by shortening TE and by increasing $N$ the effect of T1-relaxation can be reduced. The good T2-like contrast achieved in our study suggests that by using proper imaging parameters the role of $\mathrm{T} 1$ relaxation may become insignificant. At high field strengths the T1 relaxation times of tissues are longer and the contrast should be even more Tl $\rho$ weighted.

\subsubsection{Comparison of tumor CNR between SL and T2-weighted sequences}

We compared the tumor contrast between opposed-phase multiple slice SL-GRE sequence and T2-weighted SE sequence in head and neck tumor imaging. With our imaging parameters no significant differences in the overall CNRs were observed between these two sequences. However, CNR between tumors and muscles was significantly better with T2 weighted technique, whereas the CN Rs between tumors and salivary gland tissue were markedly better with the SL technique. The greater signal intensity suppression of salivary gland tissue and better contrast on SL-GRE images are probably due to fat/water cancellation with the chosen TE of $30 \mathrm{~ms}$.

\subsubsection{Salivary gland opposed-phase multiple slice SL imaging}

The fat content of salivary glands increases with aging and varies a lot between individuals (Scott et al. 1987). This poses a challenge for salivary gland tumor imaging and for contrast optimization.

Theoretically, in the salivary gland imaging employing opposed-phase SLGRE technique could be one solution for this problem. The greater signal suppression of fatty glandular tissue should enhance the conspicuousness of tumors. This is supported with our results of better contrast with opposed-phase SL imaging compared to T2-weighted imaging. With the SL imaging all tumors adjacent to salivary gland were hyperintense and easy to detect. 


\subsubsection{Comparison between SL- and T2-weighted imaging techniques}

The T2 contrast is generated within long TEs, which allow the spins to dephase through various mechanisms, and cause harmful artifacts to images. In T1p imaging the spins are locked during the contrast evolution time. The phase coherence of spins is maintained and susceptibility artifacts are reduced in comparison to T2-weighted imaging, especially at high field strengths (Engelhardt and Johnsson 1996). In the SL technique the optimization of the signal collection according to the maximum CNR is more convenient, because major part of the contrast is generated separately from data collection during application of the locking pulses.

The reduced motion sensitivity of SL imaging compared to SE imaging is demonstrated on SL images (Figs 7a and $8 b$ and 9a) where CSF appears bright. On the corresponding SE images (Figs $7 b$ and $8 c$ and $9 b$ ) CSF is dark. This is because $S L$ image is generated using gradient echo with short TE, and the flowing spins are not dephased in such extent as in the SE sequence with a long TE. In addition, the selective refocusing pulse in the SE sequence does not affect all the flowing spins which have received the earlier selective excitatory pulse. Thus, the signal intensity received from flowing spins is greatly reduced in SE imaging compared to multiple slice SL imaging.

\subsubsection{Fat}

The combination of relatively short $\mathrm{T} 1$ and long $\mathrm{T} 1 \rho$ relaxation times explains the brightness of fat on multiple slice SL images (Figs 7-9). As discussed above, the long T1 $\rho$ observed with the SL technique can be probably explained by maintained phase coherence during the locking pulse. In study IV a satisfactory contrast between tumor and fat tissue was achieved on SL images. The bright fat produced especially good contrast to low-signal-intensity malignant tumors. Adding the fat suppression technique to SL imaging is al so technically possible. For evaluation of benign tumors and also lymph nodes, the combination of SL imaging and fat suppression technique is likely to provide better contrast.

\subsubsection{GRE- versus SE-SL imaging}

In patient work (IV) we used the multiple slice SL-GRE technique instead of SLSE technique for two reasons. First, with GRE technique, the opposed-phase technique could be used with its benefits in fat containing tissues such as salivary glands, as discussed above. Another advantage of GRE over SE technique is that higher CN Rs can be obtained when TE is short. TE being short the maximum effective sampling time is longer (bandwidth is narrower) with GRE than with SE technique because there is no refocusing pulse, and consequently CN Rs should be higher with GRE vs. SE technique.

The GRE sequences are known to be subject to susceptibility artifacts which can destroy the image quality especially in the skull base or in the mouth region when metal objects are present. By using the combination of short locking pulses and short TE the phase coherence of spins is better maintained and susceptibility 
artifacts have minor effect on image quality (Fig. 7). This is especially important at higher field strengths where susceptibility artifacts pose a greater problem for M RI imaging (Engelhardt and Johnsson 1996).

\section{Head and neck tissue characterization (I, II)}

\subsection{T2 evaluation versus SL and MT effects}

Conventional MRI has been widely used for characterization of head and neck tumors and associated lymphadenopathy with unsatisfactory results (Dooms et al. 1985, Vogl et al. 1990, Sigal et al. 1992, Freling et al. 1992 and Takashima et al. 1993, Wagner-M anslau et al. 1994). In salivary gland imaging, it has been reported that highly malignant parotid tumors, which are relatively uncommon, are characterized by low signal intensity on both T1- and T2-weighted images (Som and Biller 1989, Takashima et al. 1997). In two studies with large number of patients (Freling et al. 1992 and Takashima et al. 1993) the T2-weighted signal intensity characteristics were found to be poor diagnostic criteria in predicting the histological nature of parotid and other head and neck tumors. M ost of the parotid tumors, either benign or malignant, were characterized on M R images as having high T2-weighted signal intensities when compared to normal salivary gland tissue.

In study I, our results are in agreement with these previous works. We found the evaluation of qualitative T2-weighted signal intensity characteristics unreliable criteria in differentiating benign and malignant head and neck tumors. However, in study II, the accuracies with T2-weighted signal intensity evaluation and with T1p or MT techniques were essentially the same. In study II, we had three high grade and three low grade malignancies with low signal intensity compared to parotid gland, and the qualitative $\mathrm{T} 2$ signal intensity evaluation was more reliable than in the previous studies (Freling et al. 1992 and Takashima et al. 1993). In study II, there were three patients with low-grade malignancies, whose parotid glands had probably undergone fatty degeneration. Thus, the T2 signal intensity of parotid glands was exceptionally bright in these patients, which explains the right positive findings with $\mathrm{T} 2$ evaluation for those low-grade malignancies.

\subsection{Tumor characterization with MT and SL effects}

MT imaging has been used for tissue characterization of brain and head and neck tumors both at $0.1 \mathrm{~T}$ and 1.5 T. (Lundbom 1992, Yousem et al. 1994, Kurki et al. 1996 and Takashima et al. 1999). O ur findings with M T effects of tissues and different tumors are congruent with those reported by Yousem and co-workers (1994). However, in the study by Takashima and associates (1999) the MT effects obtained were clearly smaller than in our study or in the study by Yousem et al. (1994). In addition to differences in MT pulse parameters, the explanation for smaller MT effects in their study probably resulted from T1-weighted sequence with antagonistic $\mathrm{T} 1$ effects. 
Yousem and co-workers (1994) demonstrated a highly significant difference in MT effects of benign versus malignant neoplasms. However, there were 10 benign tumors (44 malignancies) and only three parotid tumors which probably explained the statistically stronger difference between benign and malignant neoplasms found in their study compared to our work. On the basis of Lundbom's (1992) experience investigators hypothesized that M T effects would be elevated in malignant, hypercellular, high grade neoplasms because of greater degree of macromolecular cell wall protein interactions in these lesions. In contrast to Lundbom's results in brain tumors, Yousem and colleges (1994) could not find any correlation between $\mathrm{MT}$ effects and different histologic findings in malignancies.

With our imaging parameters at $0.1 \mathrm{~T}$ an SL effect below 0.48 and an M T effect below 0.32 appeared to be a good predictor of a benign tumor. There was only one squamous cell carcinoma which was a false negative and had very low SL effect and MT effect values. In histologic examination it was a highly necrotic tumor, with high T2-weighted signal intensity and the necrosis is the probable explanation for the low effects noticed (Freling et al. 1993). Acinic cell carcinoma, also known as an acinocellular tumor is a semi malign, highly differentiated tumor, which may sometimes metastasize (Seifert 1992). We had two acinocellular neoplasms. The first one was histologically benign and regarded as an acinocellular tumor. The other one had metastasized and showed histologically malignant features. It was diagnosed as an acinic cell carcinoma. The histologically benign tumor had low SL and MT effects and the malignant tumor had high SL and MT effects.

One explanation for higher SL and MT effects in malignancies could be the amount of certain cytoplasmic interfilament proteins. The malignant tumors in our series contained more lesions of epithelial origin and thus they express more cytokeratins than benign ones. However, in the work by Yousem et al. (1994) MT effect did not correlate with the keratin formation in squamous cell carcinomas. Thus, the possible role of cytokeratins and their subtypes in SL and MT effects must be further studied.

High SL or MT effects were not reliable indicators of malignancy because there was overlapping between major salivary gland infections, some benign tumors and malignancies. Except for one mucocele, all benign tumors that showed high effects where major salivary gland tumors. The salivary gland tissue had high SL and MT effects; consequently it is not surprising for glandular infections to have high values. Pleomorphic adenoma is marked by great histologic diversity that accounts for both the high and low effects noticed (Som and Brandwein 2003). Serous and mucous fluid filled spaces common in pleomorphic adenomas probably produce the low effect values, whereas more solid mucous spaces and fibrous stroma explain the high effects. In a previous study (Takashima et al. 1999), there were also two wrong positive pleomorphic adenomas with high M T effects. Mucoceles contain mucus which may be rich in high molecular weight macromolecules and exhibit cross-linking explaining the high effects noticed (Som et al. 1989). This was supported with our case of mucocele that showed low T2 signal intensity suggesting elevated protein concentrations. 
Outside the glandular area the differentiation between benign and malignant lesions seemed to be much more reliable. The vascular malformations and tumors have been characterized as having high signal intensity pattern on T2weighted images (Becker and Kurt 1999). In the work by Yousem and colleges (1994) all three vascular tumors, 2 hemangiopericytomas and one hemangioma, had low MT effects. Our study was in agreement with these previous observations, and we had four vascular benign tumors, two paragangliomas and two angiofibromas with low SL and M T effects.

\subsection{Correlation and efficiency of SL and MT techniques}

We found a strong correlation between SL and M T effects in the head and neck tumors. This is in agreement with the suggestion that M T plays a dominant role in the $T 1 \rho$ relaxation observed with the SL technique in protein rich tissues (Brown and Koenig 1992). Assuming the dominant role of MT in T1p relaxation, the on resonance $S L$ technique seems to be more efficient in the generation of MT based contrast than conventional MT technique with off-resonance saturation pulses. The efficiency of SL technique was demonstrated in study I, where the SL effects were stronger than the MT effects in all tissues despite of using shorter TL than Toff. This is based on the following facts. The T1p relaxation times of protein rich tissues are in general considerably shorter than time constants during the off-resonance saturation pulse in the M T sequence (Komu 1992, Kajander et al. 1996, Virta et al. 1998a, Koskinen et al. 1999). The equilibrium value which the magnetization of tissues approaches during the locking pulse of a $\mathrm{SL}$ sequence is approximately zero. In the MT technique the corresponding equilibrium value is $40-60 \%$ from the maximum magnetization.

\subsection{Single slice and multiple slice imaging techniques and tissue characterization}

In the single slice SL imaging the excitation pulse of a conventional imaging sequence is applied immediately after SL preparation pulse and there is no time for T1 relaxation to take place. In the multiple slice SL imaging T1 relaxation takes place between locking pulses and reduces $T 1 \rho$ contrast. Thus, the SL effect is also reduced and tissue discrimination potential is affected. Tissues with short T1, such as high-grade malignancies, have larger antagonist T1 effect which theoretically reduces the discrimination potential of multiple slice SL technique compared to single slice technique. At higher field strengths $T 1$ of tissues is longer and the antagonist T1 effect is smaller. Thus in theory, at high field strengths multiple slice SL imaging might have more potential for tissue discrimination. However, further studies are required to establish the tumor characterization potential of multiple slice SL imaging.

The tissue discrimination potential of SL and MT techniques in head and neck tumor imaging was evaluated in studies I and II. We performed interleaved single slice $S L$ and MT imaging instead of multiple slice imaging in order to max- 
imize the contribution of $T 1 \rho$ and MT relaxation processes to the SL or MT effect. We wanted to eliminate the effect of multiple slices and a more complicated contribution of T1 and T2 effects to the signal intensity. Interleaved imaging technique allowed us to keep the ROI area exactly the same in both images.

\subsection{References $\mathrm{MnCl}$ and fat}

The SL technique probes all relaxation mechanisms that are effective at very low field strengths, whereas the MT technique is sensitive only to macromolecule water interaction in protein rich tissues (UImer et al. 1996). Consistent with this, fat tissue and $\mathrm{MnCL}$ reference showed neither T1 $\rho$ dispersion effects nor MT effects whereas their SL effects were moderate (Table 2 and Table 2/II). We used $\mathrm{M} \mathrm{nCl}$ as an external and fat tissue as an internal reference to make sure that preparation pulses were correctly applied and their strength was reproducible. One can utilize the $\mathrm{MnCl}$ solutions to determine what frequency-offset range results in little direct saturation (or spin locking effect) for an MT experiment, and what frequency-offset range produces prominent direct saturation (UImet et al. 1996). In our studies $(\mathrm{I}, \mathrm{II})$ the $\mathrm{MT}$ effects for $\mathrm{M} \mathrm{nCl}$ were low with minor variability indicating low direct saturation effect with good reproducibility. The SL effects were also highly reproducible.

\subsection{Tlp dispersion imaging of head and neck tissues}

The potential of $T 1 \rho$ dispersion imaging to differentiate benign and malignant head and neck tumors was evaluated and compared with spin lock and magnetization transfer techniques. The diagnostic efficacy in differentiating benign and malignant head and neck tumors was approximately the same with all these techniques. However, there were distinct differences between the calculated effects of these techniques in normal tissues, such as fat and parotid gland, and also in $\mathrm{MnCl}$ reference.

Parotid gland tissue effects demonstrated great variability. There were three parotid glands with neither T1 $1 \rho$ dispersion effects nor M T effects while their SL effects were moderate. Presumably, these glands had undergone fatty degeneration which was also supported by the relatively high $\mathrm{T} 2$ signal intensity characteristics. This is probably also the explanation for lower T1 $1 \rho$ dispersion and M T effects and higher variability of glandular tissue observed in study II as compared with studies I and III. Muscle tissue showed the strongest effects with all techniques. Our results of T1p dispersion behavior of fat and muscle tissues are in agreement with previous studies at low field strengths (Tanttu et al. 1986, Virta et al. 1998a, Koskinen et al. 1999)

A T $1 \rho$ dispersion effect below 0.14 appeared to be a good predictor of a benign tumor, when our imaging parameters and a $0.1 T$ imager were used. There was only one false negative malignancy, a plasmocytoma, which had slightly lower T1 $\rho$ dispersion and MT effects than the threshold. However, the SL effect was high, suggesting a malignant tumor. This is probably due to the relatively high fat 
content seen in histologic slices of a plasmocytoma, because the tumor was infiltrating the fatty bone marrow of the skull base. Fat has neither T $1 \rho$ dispersion nor MT effect whereas its SL effect is moderate and quite near to that of a malignant tissue. A large fat content reduces T1 1 dispersion and M T effects more than it has influence on $\mathrm{SL}$ effect.

High Tlp dispersion effects were not reliable indicators of malignancy be cause chronic infections, some benign tumors and malignancies may overlap. We had one chronic sinusitis which showed high Tlp dispersion effects. Two benign tumors, an adenolymphoma and a mucocele, also showed high effects. The adenolymphoma was rich in lymphatic tissue and histologically resembled lymphomas explaining the high Tlp dispersion effects. This is in agreement with the study by Takashima and colleges (2001) where adenolymphoma also produced high MT effects. Mucoceles may contain very viscous mucus explaining the high effects (Som et al. 1989).

\section{Future prospects}

One advantage of low field strengths is the low RF absorption in tissues. In our study at $0.1 \mathrm{~T}$ the SAR values were far below the safety limits (Huurto and Toivo 2000). Therefore, the selection of imaging parameters is more convenient and may be adapted to the current clinical imaging task. SL technique has been implemented also for human imaging at higher field strengths (Dixon et al. 1996, U Imer et al. 1996, Semple et al. 1998, U Imer et al. 1998). By using suitable imaging parameters and off-resonance technique the exceeding of SAR limits has been avoided (Santyr et al. 1994). Higher SN R combined with even enhanced T1 $p$ contrast (due to longer T1 values of tissues) makes multiple slice SL technique an attractive method for tissue characterization and contrast enhancement also for high field strengths. It might be interesting to correlate SL and MT effects with long-term follow-up to see whether these effects may have any predictive value for recurrent tumors or metastases.

An interesting future application of SL imaging could be M R angiography (Azhari et al. 2001). Reduced motion sensitivity and MT like background suppression effects are an interesting combination when angiography applications are considered. MT imaging has been widely and successfully used for M RI angiography (Edelman et al. 1992). The somewhat limiting factor has been poor suppression of fat in MIP images (Finelli 1994). In SL imaging the suppression of tissues is more efficient than in $M T$, and especially fat is more effectively suppressed (study I). Thus, assessing the performance of multiple slice or 3D SL imaging in $\mathrm{MRI}$ angiography at high field strength would be interesting.

Another interesting prospect for future studies could be the combined use of $\mathrm{SL}$ effect with either MT effect or T1 $\rho$ dispersion imaging for detection of fat or paramagnetic substances in order to enhance the tissue characterization potential of M RI. M inimal MT or T1p dispersion effect coinciding with moderate SL effect would be characteristic for fat in benign tumors such as adenomas or dermoids. 
To assess the combination of SL and MT effects in detection of paramagnetic blood degradation products would also be interesting. This might be used for characterization of hemorrhagic adnexal lesions such as endometriomas.

In recent animal studies at 4.7 T (Gröhn et al. 2000, Kettunen et al. 2001) T1p and $T 1 \rho$ dispersion parameters have been shown to be sensitive indicators of irreversible cell damage in acute brain infarct with comparable results to diffusion weighted imaging. In a study by Tailor and co-workers (2003) T1p imaging was found effective in the quantitation of cerebral blood flow in rats at 4T. The authors recommended the technique to be suitable also for human perfusion studies at high fields. This suggests that T $1 p$ imaging might be used as an alternative method to the DWI and currently available perfusion methods in patients with acute brain infarct for the selection of adequate therapy measures.

\section{Clinical implications}

In head and neck tumor imaging the differentiation of early fibrosis from tumor recurrence is a particularly difficult problem. (Chong and Fan 1997, Lell et al. 2000). The high water content and hypercellularity in early fibrosis produce high signal intensity on T2 weighted images. An immature scar cannot be differentiated from tumor because both show enhancement on T1-weighted images and high signal intensity on T2-weighted images.

As we have demonstrated, the contrast on SL and MT images resembles T2weighted images. It is probable that these imaging techniques would not provide major advantages compared to T2 imaging in differentiation of tumor recurrence from early scar tissue. SL and MT effects in recurrent tumor tissue would probably be approximately on the same level as in immature scar tissue. In mature scar tissue with collagen and low water content the effect values should be higher allowing more reliable differentiation between tumor and scar tissue.

M R imaging criteria for malignant infiltration of lymph nodes are not very accurate and they are based on size criteria and the presence of necrosis (van den Brekel et al. 1990). In studies III and IV the T1 $\rho$ relaxation times of lymphoid tissue were partly overlapping with malignancies. It is probable that the microscopic infiltration of lymph nodes by cancer cells would not have much effect on respective M T or SL effect values. The necrosis of a tumor results in more liquid, less cellular sample and lower SL and MT effects as noted in study I. The necrotic lymph nodes could probably be detected by SL or MT techniques with clearly lowered effects depending on the amount of necrosis present in the lymph node. Thus, the differentiation between benign and malignant lymph nodes with SL or M T techniques would be complicated and challenging.

As it stands, the role of SL or M T imaging serves as an alternative for T2weighted imaging in the detection of tumors, and mapping the extent of the disease rather than in the identification of histologic specificity. The multiple slice $\mathrm{SL}$ imaging provides $\mathrm{T} 2$ like contrast with wide anatomical coverage and reduced motion and susceptibility sensitivity for head and neck region. Calculation of SL 
or MT effects is unspecific for tumor differential diagnosis, but low effects are highly suggestive of a benign tumor. However, the cut-off values are not constant and they depend on technical aspects and sequence parameters used. Thus, the cut-off values should be determined specifically for each MRI device and sequence protocol to be applied for head and neck tumor imaging. 


\section{CONCLUSIONS}

T $1 p$ values of tumors and normal head and neck tissues, measured at B1L of 35 $\mu \mathrm{T}$, were slightly longer than corresponding $\mathrm{T} 2$ relaxation times. The exception was fat tissue with $T 1 \rho$ relaxation time considerably longer than $T 2$ relaxation time and slightly shorter than $\mathrm{T} 1$ value.

Multiple slice opposed-phase fat/water SL-GRE technique provides image contrast comparable to SE T2 weighted imaging for head and neck tumors. The combination of short locking pulses ( $T L=10-35 \mathrm{~ms}$ ) and of short TE of $30 \mathrm{~ms}$ produces optimum CNR for clinical tumor imaging with wide anatomical coverage and reduced motion and susceptibility artifacts compared to T2 weighted imaging. The out of phase fat/water SL technique is especially advantageous in salivary gland tumor imaging.

Low T1p dispersion effects, SL effects and MT effects are characteristic of benign head and neck tumors. High effect values are not specific indicators of malignancy because the effects of chronic salivary gland infections, some benign tumors and malignancies may overlap.

A strong correlation between SL and MT effects in head and neck tumors supports the theory that MT plays a dominant role in the T1 $\rho$ relaxation observed with SL technique in protein rich tissues.

In the SL imaging we used a shorter preparation time of $T L=100 \mathrm{~ms}$ than in the M T imaging Toff $=500 \mathrm{~ms}$. Despite this the SL effects were stronger than the MT effects in all tissues measured. Hence the SL technique seems to be an effective method in generation of MT based contrast and tissue specific information in the head and neck tumors. 
This study was carried out as collaboration between the Department of Radiology, the Department of Otorhinolaryngology and the Department of Pathology, Helsinki University Central Hospital.

I wish to express my sincere gratitude to Professor Leena Laasonen, M .D. for her interest in my scientific work. I also wish to thank emeritus Professor CarlGustaf Standertskjöld -Nordenstam, M.D., the former head of the Department of Diagnostic Radiology, for providing me with the facilities to carry out this study and for his interest in my work.

I owe my deepest gratitude and respect to my supervisor, Professor Raimo Sepponen, Ph.D., "Godfather of the low field MRI in Finland", who introduced me to the fascinating, but beforehand completely unfamiliar, low field MRI and guided me throughout the years. $\mathrm{H}$ is intelligence and wide knowledge of MRI physics have been vitally important for this work; and also his great sense of humour has made our collaboration not only interesting but also very enjoyable.

I am most grateful to my supervisor Professor Hannu Aronen, M.D., Ph.D., who suggested the subject of the thesis. Our stimulating discussions with encouragement and optimism towards my study have been essential for me to finally complete this work. I recall his, sometimes a bit desperate, attempts to persuade me to get the thesis ready: "if there is only one doctor in the family and it is "a she", the situation is not good for the man's well lbeing in the long run".

I wish to thank Docent Pekka Niemi, M.D., and M arkku Komu, Ph.D., the official reviewers, for their interest and careful review of the final manuscript. Their valuable comments and constructive criticism greatly improved the text.

I owe my warmest thanks to my co-workers; we had a good team: Jukka Tanttu, Ph.D. for providing his expertise on MRI technology and physics at my disposal. Professor Timo Paavonen, M .D. for pathologic analysis and expertise. Erkki Hopsu, M.D. for providing us with the patients and for his expertise on clinical subjects. Leena Lauronen, M .D. for helping me in patient imaging and for guiding how to use computers and software. Usama Abo Ramadan, Ph.D. for teaching me MRI physics and helping me to image patients. Sakari Lukkarinen, MSc. for helping me in theoretical calculations of contrast. Juha Halavaara, M.D. for reviewing the text and for enjoyable and humorous discussions on various subjects, not least on sports.

I owe my warmest thanks to the whole personnel at the Department of "Stomach and Ear" radiology for their kind and enjoyable collaboration at daily work during these several years. My thanks go to the present and former colleagues at the department: Merja Raade, M.D., Docent Soraya Robinson, M.D., Jari Juola, M.D., Arto Kivisaari, M.D., Anneli Piilonen, M.D., M aarit Palomäki, M .D., Docent Tapio Vehmas, M .D., Docent M artti Pamilo, M .D., Pekka M ankinen, M.D. and Fredrik Koivusalo, M.D., for their pleasant collaboration and especially for their patience towards my numerous EVOs. I wish to thank Docent Kaarina 
Partanen, M.D. head of the department for her encouraging attitude towards this endlessly continuing work.

I am very grateful to the members of football team AJAKS and Singing Group Sepänkatu Singers for participating as "normal" volunteers in study III. Special recognition goes to my good friends Risto Jäntti, M.D. for his excellent calmness to lie in the tube without moving, which produced perfect images for publication; and for Reino Piironen, M Sc., for his patience to lie in the tube for more than two hours (there was some trouble with the M RI unit) without moving a finger. I recall his enthusiastic comment after an imaging session: "it was a very stimulating experience, those gradients produced ingenious thoughts into my mind".

I wish to thank the chairman of the sports club Coitos docent Ville Pettilä, $M$.D., and the other members of the club for their "loving but not so encouraging" support in our joyful and colourful "after game" meetings and for arranging various refreshing sports activities which have given me strength to go on this "long and winding road". The numerous winning awards have given me self-confidence to complete this work. I am grateful to emeritus chairman of Coitos Pekka Rikkonen, M .D., M .A., for revising the English language of the thesis.

I wish to thank the members of the singing group Sepänkatu Singers for joyful singing sessions on various occasions and celebrations. First recording session and many more concerts are still to come. Life without music (and football) would be much more boring.

I owe my warmest and longing thanks to my late parents Rauha and Olavi for their loving support in every aspect of my endeavours and accomplishments.

The deepest thanks are due to my dear family H elena, Joel and M arkus. Without Helena's example 10 years ago (her thesis) I might even never have began the research work, but I can forgive her for that. I want to thank H elena for her loving support and good sense of humour which have cheered me up in dull moments of everyday life and whenever I have struggled with the thesis. It has not always been so easy for you boys, and neither for me, to understand "why that thesis is so important". As Markus ( 6 yrs.) said to me one morning after a couple of long working days: "Is it fair, Antti that you are my father and still I have not seen you in two days?" I can promise you boys that from now on I have even more time for your sporting and other activities.

This work was supported by the Radiological Society of Finland, Cancer Organizations of Finland, Pehr O scar Klingendahl Foundation, Finnish M edical Association Duodecim, Waldemar von Frenckell's foundation, the state subsidy for University Hospitals, and research grants from Helsinki University Central Hospital.

Helsinki, September 2003
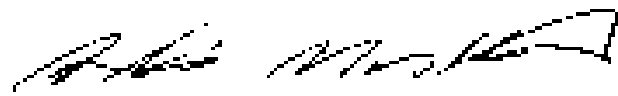

Antti Markkola 


\section{REFERENCES}

Aassar OS, Fischbein NJ, Caputo GR, Kaplan MJ, Price DC, Singer MI, Dillon WP, Hawkins RA. Metastatic head and neck cancer: role and usefulness of FDG PET in locating occult primary tumors. Radiology. 1999;210:177-181

Abo Ramadan U, Aronen HJ, Tanttu JI, Karjalainen PT, Kairemo KJ, Jaaskelainen J, Sipila $\mathrm{L}, \mathrm{H}$ akkinen AM . Improvement of brain lesion detection at $0.1 \mathrm{~T}$ by simultaneous use of Gd-DTPA and magnetization transfer imaging. Magn Reson M ed 1997;37:268-174

Adams S, Baum RP, Stuckensen T, Bitter K, Hor G. Prospective comparison of 18F-FDG PET with conventional imaging modalities (CT,M RI, US) in lymph node staging of head and neck cancer. Eur J Nucl Med 1998;25:1255-1260

Akella SV, Regatte RR, Gougoutas AJ, Borthakur A, Shapiro EM, Kneeland JB, Leigh JS, Reddy R. Proteoglycan-induced changes in T1rho-relaxation of articular cartilage at 4T. Magn Reson Med 2001;46:419-423

Anzai Y, Prince MR. Iron oxide-enhanced M R lymphography: the evaluation of cervical lymph node metastases in head and neck cancer. J Magn Reson Imaging. 1997;7:7581. Review

Aronen HJ, Ramadan UA, Peltonen TK, M arkkola AT, Tanttu JI, Jaaskelainen J, Hakkinen AM, Sepponen R. 3D spin-lock imaging of human gliomas. Magn Reson Imaging 1999;17:1001-1010

Azhari H, McKenzie CA, Edelman RR. MR angiography using spin-lock flow tagging. $M$ agn Reson M ed 2001;46:1041-1044

Balaban RS, Ceckler TL. Magnetization transfer contrast in magnetic resonance imaging. Magn Reson Q 1992;8:116-137

Becker M , Kurt A-M . Infrahyoid neck: CT and M R-imaging versus histopathology. Eur Radiol. 1999;9 (Suppl. 2) 53-68

Bilbey JH, McLoughlin RF, Kurkjian PS, Wilkins GE, Chan NH, Schmidt N, Singer J. MR imaging of adrenal masses: value of chemical-shift imaging for distinguishing adenomas from other tumors. Am J Roentgenol 1995;164:637-642

Bloch F, Hansen WW, Oackard M. Nuclear induction. Phys Rev 1946;69:127

Boorstein JM, Wong KT, Grossman RI, Bolinger L, M CGowan JC. M etastatic lesions of the brain: imaging with magnetization transfer. Radiology 1994;191:799-803

Borcard B. Field dependence of relaxation times. Nuclear magnetic relaxation dispersion. Prog Nucl M ed. 1984;8:47-54

Bottomley, PA., Hardy, CJ, Arsinger, RE, Allen-More G. A review of $1 \mathrm{H}$ nuclear magnetic resonance relaxation in pathology: are T1 and T2 diagnostic? M ed Phys 1987;14:1-37

Brown RD, Koenig SH. 1/T $1 \rho$ and low-field 1/T1 of tissue water protons arise from magnetization transfer to macromolecular solid-state broadened lines. Magn Reson Med 1992;28:145-152.

Castelijns A, van den Brekel MW. Detection of lymph node metastases in the neck: radiologic criteria. Am J Neuroradiol 2001;22:3-4

Chong VF, Fan YF. Detection of recurrent nasopharyngeal carcinoma: M R imaging versus CT. Radiology 1997;202:463-70

Conti S. Nuclear magnetic relaxation in weak fields. Application to the study of blood. Prog Nucl Med 1984;8:71-7

Crooks L, Arakawa M, Hoenninger J, Watts J, McRee R, Kaufman L, Davis PL, M argulis $A R$, DeGroot J. Nuclear magnetic resonance whole-body imager operating at 3.5 KGauss. Radiology 1982;143:169-174

Damadian R. Tumor detection by nuclear magnetic resonance. Science 1971;171:11511153 
de Bree R, Roos JC, Quak JJ, den Hollander W, van den Brekel M W, van der Wal JE, Tobi $H$, Snow GB, van Dongen GA. Clinical imaging of head and neck cancer with technetium-99m-labeled monoclonal antibody E48 IgG or F(ab')2. J Nucl M ed 1994;35:775783

Dixon WT, Oshinski JN, Trudeau JD, Arnold BC, Pettigrew RI. Myocardial suppression in vivo by spin locking with composite pulses. Magn Reson Med 1996;36:90-94

Dooms GC, H ricak H, M oseley M E, Bottles K, Fisher M, Higgins CB. Characterization of lymphadenopathy by magnetic resonance relaxation times: preliminary results. Radiology 1985;155:691-697

D ousset V, Grossman RI, Ramer KN, Schnall MD, Young LH, Gonzalez-Scarano F, Lavi E, Cohen JA. Experimental allergic encephalomyelitis and multiple sclerosis: lesion characterization with magnetization transfer imaging. Radiology 1992;182:483-491. Erratum in Radiology 1992;183:878

Duvvuri U, Reddy R, Patel SD, Kaufman JH, Kneeland JB, Leigh JS. T1rho-relaxation in articular cartilage: effects of enzymatic degradation. Magn Reson Med 1997;38:863867

Duvvuri U, Roberts DA, Leigh JS, Bolinger L. M agnetization transfer imaging of the brain: A quantitative comparison of results obtained at 1.5 and 4.0 T.J M agn Reson I maging 1999;10:527-532

Edelman RR, Ahn SS, Chien D, Li W, Goldmann A, Mantello M, Kramer J, Kleefield J. Improved time of-flight $M R$ angiography of the brain with magnetization transfer contrast. Radiology 1992;184:395-399

Edelstein WA, Bottomley PA, Hart HR, Smith LS. Signal, noise, and contrast in nuclear magnetic resonance (N M R) imaging. J Comput Assist Tomogr 1983;7:391-401

Engelhardt RT, Johnson GA. T1 rho relaxation and its application to M R histology. Magn Reson Med 1996;35:781-786

Finelli DA, Hurst GC, Gullapali RP, Bellon EM . Improved contrast of enhancing brain lesions on postgadolinium, T1-weighted spin-echo images with use of magnetization transfer. Radiology 1994;190:553-559

Flamig DP, Pierce WB, H arms SE, Griffey RH . M agnetization transfer contrast in fat-suppressed steady-state three-dimensional M R images. Magn Reson Med 1992;26:122131

Fralix TA, Ceckler TL, Wolff SD, Simon SA, Balaban RS. Lipid bilayer and water proton magnetization transfer: effect of cholesterol. Magn Reson M ed 1991;18:214-223

Freling NJ, Molenaar WM, Vermey A, M ooyaart EL, Panders AK, Annyas AA, Thijn CJ. $M$ alignant parotid tumors: clinical use of $M R$ imaging and histologic correlation. Radiology 1992;185:691-696

Fulbright R, Panush D, Sze G, Smith RC, Constable RT. M R of the head and neck: comparison of fast spin-echo and conventional spin-echo sequences. Am J Neuroradiol 1994;15:767-773

Gomori JM, Grossman RI, Goldberg HI, Zimmerman RA, Bilaniuk LT. Intracranial hematomas: imaging by high-field M R. Radiology 1985;157:87-93

Greven KM, Williams DW 3rd, McGuirt WF Sr, Harkness BA, D'Agostino RB Jr, Keyes JW $\mathrm{J} r$, Watson NE Jr. Serial positron emission tomography scans following radiation therapy of patients with head and neck cancer. Head Neck 2001;23:942-946

Gröhn OHJ, Kettunen MI, M äkelä HI, Penttonen M, Pitkanen A, Lukkarinen JA, Kauppinen RA. Early detection of irreversible cerebral ischemia in the rat using dispersion of the magnetic resonance imaging relaxation time, T1rho. J Cereb Blood Flow Metab 2000;20:1457-1466

Guo Y, Cai YQ, Cai ZL, Gao YG, An NY, Ma L, Mahankali S, Gao JH. Differentiation of clinically benign and malignant breast lesions using diffusion-weighted imaging. J M agn Reson Imaging 2002;16:172-178 
Haase A, Frahm J, Matthaei D, Hanicke W, Merboldt KD. FLASH imaging: rapid NMR imaging using low flip-angle pulses. J M agn Reson 1986;67:258-266

Hakumäki JM, Gröhn OH, Tyynela K, Valonen P, Yla-Herttuala S, Kauppinen RA. Early gene therapy-induced apoptotic response in BT4C gliomas by magnetic resonance relaxation contrast $\mathrm{T} 1$ in the rotating frame. Cancer GeneTher 2002;9:338-345

Halavaara JT, Lamminen AE, Bondestam S, Sepponen RE, Tanttu JI. Spin lock magnetic resonance imaging in the differentiation of hepatic haemangiomas and metastases. Br J Radiol 1995;68:1198-1203

Halavaara JT, Sepponen RE, Lamminen AE, Vehmas T, Bondestam S. Spin lock and magnetization transfer MR imaging of local liver lesions. Magn Reson Imaging 1998;16:359-364

Heaney MD and Ehman RL. Interpretation of magnetic resonance images. In: Berquist $\mathrm{TH}$, ed. M RI of the musculoskeletal system. 4th ed. Philadel phia, Lippincott Williams and Wilkins 2001;30-57

Hendrick RE. Image contrast and noise. In Stark DD, Bradley WG Jr, eds. M agnetic resonance imaging. 3rd ed. St Louis, M osby, 1999;43-67

H ollis DP, Economou JS, Parks LC, Eggleston JC, Saryan LA, Czeister JL. Nuclear magnetic resonance studies of several experimental and human malignant tumors. Cancer Res 1973;33:2156-2160

Holodny $\mathrm{Al}$, Ollenschlager $\mathrm{M}$. Diffusion imaging in brain tumors. Neuroimaging Clin $\mathrm{N}$ Am 2002;12:107-124

H onigschnabl S, Gallo S, Niederle B, Prager G, Kaserer K, Lechner G, H einz-Peer G. How accurate is $M R$ imaging in characterisation of adrenal masses: update of a long-term study. Eur J Radiol 2002;41:113-122

Huurto $L$ and Toivo T. Magneettitutkimusten turvallisuus ja suositukset. Magneettitutkimukset ja niiden turvallisuus. Lääkelaitoksen julkai susarja 1/2000;10-15, Helsinki

Iino M. Transition from Lorentzian to Gaussian line shape of magnetization transfer spectrum in bovine serum albumin solutions. M agn Reson M ed 1994;32:459-463

I maoka I, Sugimura K, O kizuka H, I wanari O, Kitao M , Ishida T. Ovarian cystic teratomas: value of chemical fat saturation magnetic resonance imaging. $\mathrm{Br} J$ Radiol 1993;66:994-997

Inch WR, M cCredie JA, Knispel RR, Thompson RT, Pintar M M. Water content and proton spin relaxation time for neoplastic and non-neoplastic tissues from mice and humans. J Natl Cancer Inst 1974;52:353-356

Jones GP. Spin-lattice relaxation in the rotating frame: weak-collision case. Phys. Rev. 1966;148:332-335

Just $M$, Thelen $M$. Tissue characterization with $T 1, T 2$, and proton density values: results in 160 patients with brain tumors. Radiology 1988;169:779-785

Kahn CE Jr, Perera SD, Sepponen RE, Tanttu JI, Tierala EK, Lipton MJ. Magnetization transfer imaging of the abdomen at $0.1 \mathrm{~T}$ : detection of hepatic neoplasms. Magn Reson Imaging; 1993;11:67-71

Kajander S, Komu M, Niemi P, Kormano M. Determination of saturation transfer parame ters of human tissues in vivo. M agn Reson Imaging 1996;14:413-417

Kamman RL, Go KG, M uskiet FA, Stomp GP, Van Dijk P, Berendsen HJ. Proton spin relaxation studies of fatty tissue and cerebral white matter. Magn Reson Imaging. 1984;2:211-220

Katayama M, M asui T, Kobayashi S, Ito T, Sakahara H, Nozaki A, Kabasawa H. Diffusionweighted echo planar imaging of ovarian tumors: is it useful to measure apparent diffusion coefficients? J Comput Assist Tomogr 2002;26:250-256

Kettunen MI, Gröhn OH, Penttonen M, Kauppinen RA. Cerebral T1rho relaxation time increases immediately upon global ischemia in the rat independently of blood glucose and anoxic depolarization. Magn Reson Med 2001;46:565-572 
Kettunen MI, Gröhn OH, Silvennoinen MJ, Penttonen M, Kauppinen RA. Effects of intracellular $\mathrm{pH}$, blood, and tissue oxygen tension on T1rho relaxation in rat brain. Magn Reson Med 2002;48:470-477

Kim DK, Ceckler TL, Hascall VC, Calabro A, Balaban RS. Analysis of water-macromolecule proton magnetization transfer in articular cartilage. Magn Reson Med 1993;29:211-215

Knispel RR, Thompson RT, Pintar M M. Dispersion of proton spin-lattice relaxation in tissue. J Magn Res 1974;14:44-51

Koenig SH, Brown RD 3rd, Adams D, Emerson D, Harrison CG. Magnetic field dependence of 1/T 1 of protons in tissue. Invest Radiol 1984;19:76-81

Koenig SH. Cholesterol of myelin is the determinant of gray-white contrast in M RI of brain. Magn Reson Med 1991;20:285-291

Koenig SH, Brown RD III, Ugolini R. A unified view of relaxation in protein solution and tissue, including hydration and magnetization transfer. Magn Reson Med 1993;14:7783

Komiyama M, Yagura H, Baba M , Yasui T, H akuba A, Nishimura S, Inoue Y. M R imaging: possibility of tissue characterization of brain tumors using T1 and T2 values. Am J Neuroradiol 1987;8:65-70

Komu M. Analysis of longitudinal relaxation rate constants from magnetization transfer M R images of human tissues at 0.1 T. Magn Reson Imaging 1992;10:35-40

Koskinen SK, Yla-Outinen H, Aho HJ, Komu ME. Magnetization transfer and spin lock M R imaging of patellar cartilage degeneration at 0.1 T. Acta Radiol 1997;38:10711075

Koskinen SK, Virta AM, Niemi PT, Kajander SA, Komu ME. T1rho dispersion of rat tissues in vitro. M agn Reson Imaging 1999;17:1043-1047

Kucharczyk W, M acdonald PM, Stanisz GJ, Henkelman RM. Relaxivity and magnetization transfer of white matter lipids at M R imaging: importance of cerebrosides and $\mathrm{pH}$. Radiology 1994;192:521-529

Kurki TJ, Niemi PT, Lundbom N. Gadolinium-enhanced magnetization transfer contrast imaging of intracranial tumors. J M agn Reson Imaging 1992;2:401-406

Kurki T, Lundbom N, Valtonen S. Tissue characterisation of intracranial tumours: the value of magnetisation transfer and conventional M RI. Neuroradiology 1995;37:515521

Kurki T, Lundbom N, Komu M, Kormano M. Tissue characterization of intracranial tumors by magnetization transfer and spin-lattice relaxation parameters in vivo. J Magn Reson Imaging 1996;6:573-579

Lam WW, Poon WS, Metreweli C. Diffusion M R imaging in glioma: does it have any role in the pre-operation determination of grading of glioma? Clin Radiol 2002;57:219225

Lamminen AE, Tanttu JI, Sepponen RE, Pihko H, Korhola OA. T 1rho dispersion imaging of diseased muscle tissue. Br J Radiol 1993;66:783-787

Lauterbur PC. Image formation by induced local interactions: examples employing nuclear magnetic resonance. Nature 1973;242:190-191

Lell M, Baum U, Greess H, Nömayr A, Nkenke E, Koester M, Lenz M , Bautz W. Head and neck tumors: imaging recurrent tumor and post-therapeutic changes with $C T$ and M RI. Eur J Radiol 2000;33:239-247

Lenz M, Greess H, Dobritz M, Kersting-Sommerhoff B. Methods: M RT. Eur J Radiol 2000;33:178- 184

Lewin JS, Curtin HD, Ross JS, Weissman JL, O buchowski NA, Tkach JA. Fast spin-echo imaging of the neck: comparison with conventional spin-echo, utility of fat suppression, and evaluation of tissue contrast characteristics. Am J Neuroradiol 1994;15:1351-1357 
Loevner LA, Grossman RI, Cohen JA, Lexa FJ, Kessler D, Kolson DL. M icroscopic disease in normal-appearing white matter on conventional $M R$ images in patients with multiple sclerosis: assessment with magnetization-transfer measurements. Radiology 1995;196:511-515

Lundbom N, Brown RD 3rd, Koenig SH, Lansen TA, Valsamis M P, Kasoff SS. Magnetic field dependence of $1 / T 1$ of human brain tumors. Correlations with histology. Invest Radiol. 1990;25:1197-1205

Lundbom N. Determination of magnetization transfer contrast in tissue: an M R imaging study of brain tumors. Am J Roentgenol 1992;159:1279-1285

M andelblatt SM, Braun IF, Davis PC, Fry SM , Jacobs LH, Hoffman JC Jr. Parotid masses: M R imaging. Radiology 1987;163:411-4

McGowan JC, Leigh JS Jr. Selective saturation in magnetization transfer experiments. Magn Reson Med 1994;32:517-522

McGuirt WF, Greven K, Williams D 3rd, Keyes JW Jr, Watson N, Cappellari JO, Geisinger KR. PET scanning in head and neck oncology: a review. H ead Neck 1998;20:208-215

Mehta RC, Pike GB, Enzmann DR. Improved detection of enhancing and nonenhancing lesions of multiple sclerosis with magnetization transfer. Am J Roentgenol 1995;16:1771-1778

M ittl RL Jr, Gomori JM , Schnall MD, Holland GA, Grossman RI, Atlas SW. M agnetization transfer effects in M R imaging of in vivo intracranial hemorrhage. Am J Neuroradiol 1993; 14:881-891

M lynarik V, Trattnig S, Huber M, Zembsch A, Imhof $\mathrm{H}$. The role of relaxation times in monitoring proteoglycan depletion in articular cartilage. J Magn Reson Imaging 1999; 10:497-502

Moran PR, Hamilton CA. Near-resonance spin-lock contrast. Magn Reson Imaging 1995;13:837-846

Mukherji SK, Fischbein NJ, Castelijns JA. New imaging techniques. In Som PM, Curtin HD, eds. Head and neck imaging. 4th ed. St Louis, M osby, 2003;2294-2322

Mulkern RV, Patz S, Brooks M, M etcalf DC, Jolesz FA. Spin-lock techniques and CPM G imaging sequences: a critical appraisal of T1p contrast at $0.15 \mathrm{~T}$. M agn Reson Imaging 1989; 7:437-444

M äkelä HI, Kettunen MI, Gröhn OH, Kauppinen RA. Quantitative T(1rho) and magnetization transfer magnetic resonance imaging of acute cerebral ischemia in the rat. J Cereb Blood Flow M etab 2002;22:547-558

Namimoto T, Yamashita Y, Sumi S, Tang Y, Takahashi M . Focal liver masses: characterization with diffusion-weighted echo-planar M R imaging. Radiology 1997;204:739-744

O kumura A, Takenaka K, N ishimura Y, Asano Y, Sakai N, Kuwata K, Era S. The characterization of human brain tumor using magnetization transfer technique in magnetic resonance imaging. Neurol Res 1999;21:250-254

Outwater E, Schnall M D, Braitman LE, Dinsmore BJ, Kressel HY. Magnetization transfer of hepatic lesions: evaluation of a novel contrast technique in the abdomen. Radiology $1992 ; 182: 535-540$

Purcell EM, Torrey HC, Pound RV. Resonance absorption by nuclear magnetic moments in a solid. Phys Rev 1946;69:37-38

Ross M R, Schomer DF, Chappell P, Enzmann DR. M R imaging of head and neck tumors: comparison of $\mathrm{T} 1$-weighted contrast-enhanced fat-suppressed images with conventional T2-weighted and fast spin-echo T2-weighted images. Am J Roentgenol 1994;163:173-178

Santyr GE, Henkelman RM, Bronskill MJ. Spin locking for magnetic resonance imaging with application to human breast. M agn Reson Med 1989;12:25-37

Santyr GE, Fairbanks EJ, Kelcz F, Sorenson JA. Off-resonance spin locking for M R imaging. Magn Reson Med 1994;32:43-51 
Scott J, Flower EA, Burns J. A quantitative study of histological changes in the human parotid gland occurring with adult age. J Oral Pathol 1987;16:505-510

Seifert G. H istopathology of malignant salivary gland tumours. Eur J Cancer B Oral Oncol 1992;28B:49-56

Sharafuddin MJ, Diemer DP, Levine RS, Thomasson JL, Williams AL. A comparison of M R sequences for lesions of the parotid gland. Am J Neuroradiol 1995;16:1895-1902

Schick F, Miller S, Hahn U, Nagele T, Helber U, Stauder N, Brechtel K, Claussen CD. Fat- and water-selective MR cine imaging of the human heart: assessment of right ventricular dysplasia. Invest Radiol 2000;35:311-318

Semple SI, Redpath TW, McKiddie FI, Waiter GD. Comparison of four magnetization preparation schemes to improve blood-wall contrast in cine short-axis cardiac imaging. Magn Reson Med 1998;39:291-299

Sepponen RE, Pohjonen JA, Sipponen JT, Tanttu JI. A method for T1 $1 \rho$ imaging. J Comput Assist Tomogr 1985;1007-1011

Sepponen RE, Tanttu JI, Suramo I. T1rho and T1rho dispersion imaging in patients with cerebral infarction. Book of Abstracts, 5th SM RM , 1986, M ontreal

Sepponen RE, Tanttu JI, Foust RJ, Kinnunen E. T1rho dispersion imaging in multiple sclerosis at 0.1T. Book of Abstracts, 9th SM RM , 1990, N ew York

Sepponen RE. Rotating frame and magnetization transfer. In: Stark DD, Bradley WG Jr, eds. Magnetic resonance imaging. 2nd ed. St Louis, M osby, 1992;204-218

Sepponen RE, Korhola O, Lamminen AE, Tanttu JI. Multiple slice spin lock sequence - an effective method for T1 $\rho$ imaging. In: Book of Abstracts, Twelveth Annual M eeting of the Society of M agnetic Resonance in M edicine, SM RM ; 1993;1218, N ew York

Sepponen RE. Low-field M R imaging-development in Finland. Acta Radiol 1996;37:446454

Sigal R, M onnet O, de Baere T, M icheau C, Shapeero LG, Julieron M, Bosq J, Vanel D, Piekarski JD, Luboinski B. Adenoid cystic carcinoma of the head and neck: evaluation with MR maging and clinical-pathologic correlation in 27 patients. Radiology 1992;184:95-101

Sigal R, Vogl T, Casselman J, Moulin G, Veillon F, Hermans R, Dubrulle F, Viala J, Bosq J, M ack M, Depondt M , M attelaer C, Petit P, Champsaur P, Riehm S, Dadashitazehozi $Y$, de Jaegere T, M archal G, Chevalier D, Lemaitre L, Kubiak C, Helmberger R, Halimi P. Lymph node metastases from head and neck squamous cell carcinoma: M R imaging with ultrasmall superparamagnetic iron oxide particles (Sinerem MR) - results of a phase-III multicenter clinical trial. Eur Radiol 2002;12:1104-1113

Som PM , Biller HF. High-grade malignancies of the parotid gland: identification with M R imaging. Radiology 1989;173:823-826

Som PM, Dillon WP, Fullerton GD, Zimmerman RA, Rajagopalan B, M arom Z. Chronically obstructed sinonasal secretions: observations on T1 and T2 shortening. Radiology 1989;172:515-520

Som PM, Brandwein MS. Salivary glands: anatomy and pathology. In Som PM, Curtin HD, eds. Head and neck imaging. 4th ed. St Louis, M osby, 2003;2005-2133

Sorensen AG, Buonanno FS, Gonzalez RG, Schwamm LH, Lev M H, Huang-Hellinger FR, Reese TG, Weisskoff RM, Davis TL, Suwanwela N, Can U, Moreira JA, Copen WA, Look RB, Finklestein SP, Rosen BR, Koroshetz WJ. Hyperacute stroke: evaluation with combined multisection diffusion-weighted and hemodynamically weighted echoplanar M R imaging. Radiology 1996;199:391-401

Sugahara T, Korogi Y, Kochi M, I kushima I, Shigematu Y, Hirai T, Okuda T, Liang L, Ge Y, KomoharaY, Ushio Y, Takahashi M. Usefulness of diffusion-weighted M RI with echoplanar technique in the evaluation of cellularity in gliomas. J Magn Reson Imaging 1999;9:53-60 
Tailor DR, Roy A, Regatte RR, Charagundla SR, M CLaughlin AC, Leigh JS, Reddy R. Indirect 170-magnetic resonance imaging of cerebral blood flow in the rat. $M$ agn Reson Med 2003;49:479-487

Takashima S, Noguchi Y, Okumura T, Aruga H, Kobayashi T. Dynamic M R imaging in the head and neck. Radiology 1993;189:813-821

Takashima S, Sone S, Takayama F, M aruyama Y, Hasegawa M, H orii A, Yoshida J. Assessment of parotid masses: which MR pulse sequences are optimal? Eur J Radiol 1997;24:206-215

Takashima S, Takayama F, Wang Q, Kawakami S, Saito A, Sone S. Head and neck lesions: determination of an optimal MT technique for prediction of malignancies. Invest Radiol 2000;35:244-252

Takashima S, Wang J, Takayama F, M omose M , M atsushita T, Kawakami S, Saito A, Ishiyama T. Parotid masses: prediction of malignancy using magnetization transfer and M R imaging findings. Am J Roentgenol 2001;176:1577-1584

Tanttu JI, Sepponen RE, Sipponen JT, Heiskanen J. Tissue parameters T1, T2, T1rho and T1 rho dispersion measured at $0.02 \mathrm{~T}$. Book of abstracts, 5th SM RM , 1986, M ontreal

Tanttu JI, Sepponen RE, Lipton MJ, Kuusela T. Synergistic enhancement of M RI with Gd-DTPA and magnetization transfer. J Comput Assist Tomogr 1992;16:19-24

Tanttu JI. Magnetic resonance imaging methods for improvement of tissue contrast especially applicable at low magnetic fields. Helsinki University of Technology applied electronics laboratory. Series B: research reports B5, 1999

Tomiak M M, Rosenblum JD, Prager JM, Metz CE. Magnetization transfer: a potential method to determine the age of multiple sclerosis lesions. Am J Neuroradiol 1994;15:1569-1574

Uchida N, Sugimura K, Kajitani A, Yoshizako T, Ishida T. M R imaging of vertebral metastases: evaluation of fat saturation imaging. Eur J Radiol 1993;7:91-94

Ulmer JL, Mathews VP, Hamilton CA, Elster AD, Moran PR. Magnetization transfer or spin-lock? An investigation of off-resonance saturation pulse imaging with varying frequency offsets. Am J Neuroradiol 1996;17:805-819

Ulmer JL, Logani SC, Mark LP, Hamilton CA, Prost RW, Garman JN. Near-resonance saturation pulse imaging of the extraocular muscles in thyroid-related ophthalmopathy. Am J Neuroradiol 1998;19:943-950

Ulmer JL, Mathews VP, Hamilton CA, Elster DA. Magnetization transfer imaging of the brain. In Stark DD, Bradley WG J r, eds. M agnetic resonance imaging. 3rd ed. St Louis, Mosby, 1999;1539- 1553

van den Brekel M W, Stel HV, Castelijns JA, Nauta JJ, van der Waal I, Valk J, Meyer CJ, Snow GB. Cervical lymph node metastasis:assessment of radiologic criteria. Radiology 1990;177:379-384

van Buchem MA, Steens SC, Vrooman HA, Zwinderman AH, McGowan JC, Rassek M, Engelbrecht $\mathrm{V}$. Global estimation of myelination in the developing brain on the basis of magnetization transfer imaging: a preliminary study. Am J Neuroradiol 2001;22:762-766

van Houten VM, Tabor MP, van den Brekel MW, Denkers F, Wishaupt RG, Kummer JA, Snow GB, Brakenhoff $\mathrm{RH}$. Molecular assays for the diagnosis of minimal residual head-and-neck cancer: methods, reliability, pitfalls, and solutions. Clin Cancer Res 2000;6:3803-3816

Virta A, Komu M, Kormano M, Lundbom N. Magnetization transfer in protein solutions at $0.1 \mathrm{~T}$ : dependence on concentration, molecular weight, and structure. Acad Radiol 1995;2:792-798.

Virta A, Kormano M , Paranko J. M agnetization transfer of pure DNA and purified sperm nuclei. M AGM A 1996;4:135-8. 
Virta A, Komu M, Kormano M. T1rho of protein solutions at very low fields: dependence on molecular weight, concentration, and structure. M agn Reson M ed. 1997;37:53-57

(a) Virta A, Komu M, Lundbom N, Kormano M. T1 rho M R imaging characteristics of human anterior tibial and gastrocnemius muscles. Acad Radiol 1998;5:104-110

(b) Virta A, Komu M, Lundbom N, Jaaskelainen S, Kalimo H, Airio A, Alanen A, Kormano M . Low field T1rho imaging of myositis. M agn Reson Imaging 1998;16:385- 391

Vogl TJ, Dresel SH, Spath M, Grevers G, Wilimzig C, Schedel HK, Lissner J. Parotid gland: plain and gadolinium-enhanced M R imaging. Radiology 1990;177:667-674

Wagner-M anslau C, Lukas P, Herzog M, Kau R, Becker K. M RI and proton-N M R relaxation times in diagnosis and therapeutic monitoring of squamous cell carcinoma. Eur Radiol 1994;4:314-323

Wang J, Takashima S, Takayama F, Kawakami S, Saito A, M atsushita T, M omose M, Ishiyama T. Head and neck lesions: characterization with diffusion-weighted echo-planar M R imaging. Radiology 2001;220:621-630

Whittall KP, MacKay AL, Li DK, Vavasour IM, Jones CK, Paty DW. Normal-appearing white matter in multiple sclerosis has heterogeneous, diffusely prolonged $\mathrm{T}(2)$. M agn Reson Med 2002;47:403-408

Williamson DS, Mulken RV, Jakab PD, Jolesz FA. Coherence transfer by isotropic mixing in Carr-Purcell-M eiboom-Gill imaging: implications for the bright fat phenomenon in fast spin-echo imaging. M agn Reson M ed 1996;35:506-513

Wolff SD, Balaban RS. Magnetization transfer contrast (M TC) and tissue water proton relaxation in vivo. Magn Reson Med 1989;0:135-144

Wolff SD, Chesnick S, Frank JA, Lim KO, Balaban RS. Magnetization transfer contrast: M R imaging of the knee. Radiology 1991; 179:623-628.

Wong KT, Grossman RI, Boorstein JM , Lexa FJ, M cGowan JC. Magnetization transfer imaging of periventricular hyperintense white matter in the elderly. Am J Neuroradiol 1995;16:253-258

Yonetsu K, Sumi M, Izumi M, Ohki M, Eida S, Nakamura T. Contribution of doppler sonography blood flow information to the diagnosis of metastatic cervical nodes in patients with head and neck cancer: assessment in relation to anatomic levels of the neck. Am J N euroradiol 2001;2:163-169

Young IR, Burl M, Clarke GJ, Hall AS, Pasmore T, Collins AG, Smith DT, Orr JS, Bydder GM , Doyle FH, Greenspan RH, Steiner RE. Magnetic resonance properties of hydrogen: imaging the posterior fossa. Am J Roentgenol 1981;137:895-901

Yousem DM, M ontone KT, Sheppard LM , Rao VM, Weinstein GS, Hayden RE. Head and neck neoplasms: magnetization transfer analysis. Radiology 1994;192:703-707

Zhong JH, Gore JC, Armitage IM. Relative contributions of chemical exchange and other relaxation mechanisms in protein solutions and tissues. Magn Reson Med 1989;11:295-308

Zhong JH, Gore JC, Armitage IM. Quantitative studies of hydrodynamic effects and cross-relaxation in protein solutions and tissues with proton and deuteron longitudinal relaxation times. M agn Reson Med 1990;13:192-203 
ORIGINAL PUBLICATIONS 
